\title{
ARTICLE
}

\section{ERCC6L2 promotes DNA orientation-specific recombination in mammalian cells}

\author{
Xiaojing Liu ${ }^{1,2}$, Tingting Liu ${ }^{1,2}$, Yafang Shang ${ }^{1,2}$, Pengfei Dai ${ }^{1,2}$, Wubing Zhang ${ }^{3}$, Brian J. Lee $\mathbb{D}^{4}$, Min Huang ${ }^{1,2}$, Dingpeng Yang (D) $^{1,2}$, \\ Qiu Wu $\mathbb{D}^{3}$, Liu Daisy Liu ${ }^{1,2}$, Xiaoqi Zheng ${ }^{5}$, Bo O. Zhou $\mathbb{D}^{1,2}$, Junchao Dong ${ }^{6}$, Leng-Siew Yeap ${ }^{7}$, Jiazhi Hu $\mathbb{D}^{8}$, Tengfei Xiao ${ }^{9}$, Shan Zha ${ }^{4}$, \\ Rafael Casellas ${ }^{10}$, X. Shirley Liu ${ }^{11}$ and Fei-Long Meng $\mathbb{D}^{1,2}$
}

Programmed DNA recombination in mammalian cells occurs predominantly in a directional manner. While random DNA breaks are typically repaired both by deletion and by inversion at approximately equal proportions, $V(D) J$ and class switch recombination (CSR) of immunoglobulin heavy chain gene overwhelmingly delete intervening sequences to yield productive rearrangement. What factors channel chromatin breaks to deletional CSR in lymphocytes is unknown. Integrating CRISPR knockout and chemical perturbation screening we here identify the Snf2-family helicase-like ERCC6L2 as one such factor. We show that ERCC6L2 promotes double-strand break end-joining and facilitates optimal CSR in mice. At the cellular levels, ERCC6L2 rapidly engages in DNA repair through its C-terminal domains. Mechanistically, ERCC6L2 interacts with other end-joining factors and plays a functionally redundant role with the XLF end-joining factor in V(D)J recombination. Strikingly, ERCC6L2 controls orientation-specific joining of broken ends during CSR, which relies on its helicase activity. Thus, ERCC6L2 facilitates programmed recombination through directional repair of distant breaks.

Cell Research (2020) 30:732-744; https://doi.org/10.1038/s41422-020-0328-3

\section{INTRODUCTION}

Programmed DNA recombination processes, including $V(D) J$ and antibody class switch recombination (CSR), diversify lymphocyte antigen receptors for efficient adaptive immunity. ${ }^{1}$ RAG endonucleases assemble V, D and J gene segments to form variable region exons of $\mathrm{B}$ and $\mathrm{T}$ cell receptor genes, ${ }^{2}$ and activationinduced cytidine deaminase (AID) further introduces DNA lesions upstream of antibody constant region genes to switch the antibody class from IgM to other classes. ${ }^{3}$

General DNA repair pathways efficiently process immunoglobulin heavy chain gene $(I g H) \mathrm{V}(\mathrm{D}) \mathrm{J}$ and CSR lesions in B cells. Despite the many mechanistic differences, in both cases intermediate breaks are joined in an orientation-specific manner, i.e. rearrangements occur predominantly by deletion rather than by inversion. ${ }^{2,4}$ This feature increases the probability of productive rearrangements and consequently the number of peripheral lymphocytes available to fight infection. Conversely, random DNA breaks or designer endonuclease-cutting ends are typically joined both by deletion and by inversion at about equal proportions. ${ }^{5}$ RAG directional linear tracking and intrinsic properties of recombination signal sequences (RSSs) enforce orientation- specific joining of $V(D) J$ breaks. ${ }^{2,6}$ How CSR ends are processed mostly by deletion is less understood.

In mammalian cells, double-strand breaks (DSBs) are sensed by the MRN complex, which activates the ATM-dependent DSB response (DSBR) pathway. ${ }^{7}$ ATM-substrate 53BP1 and its downstream effectors prevent excessive end resection to promote nonhomologous end-joining (NHEJ) at the price of homologous recombination (HR). ${ }^{8}$ The mammalian NHEJ pathway is initiated by DSB end recognition through KU70-KU86 (KU80 in mouse) heterodimer, which further recruits nucleases and/or polymerases and finally Ligase4-XRCC4-XLF complex for repair. ${ }^{9}$ Besides the evolutionarily conserved core NHEJ factors found in all eukaryotes, several new NHEJ factors have involved in vertebrates and mammals, as exampled by the recently identified NHEJ factors PAXX and MRI/CYREN. ${ }^{10-12}$ NHEJ can function flexibly on a diverse range of DSB substrates in different chromatin contexts with its DNA-based factors (e.g. core NHEJ subunits) and chromatin factors, and specialized proteins could be involved in joining of a subset of breaks.

$V(D) J$ recombination and CSR occur within the context of topological associated domains (TADs), where loop extrusion

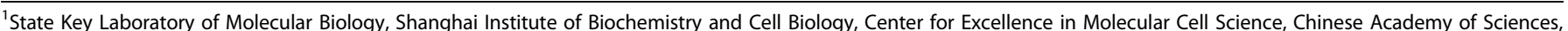

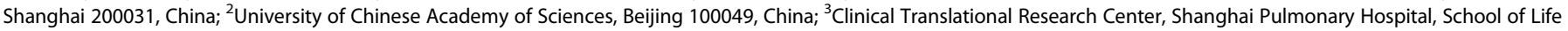

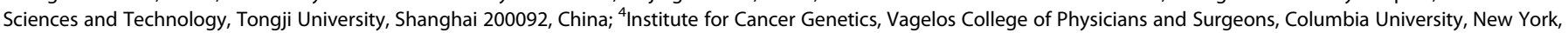

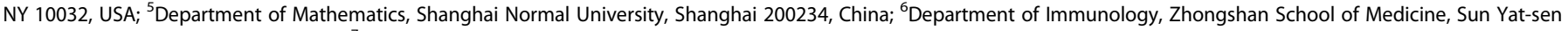

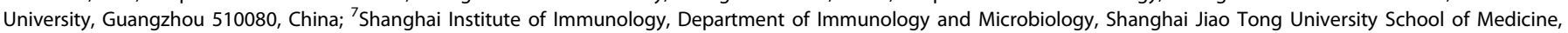

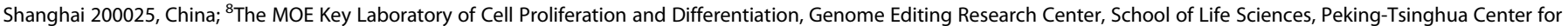

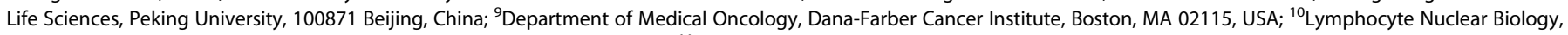

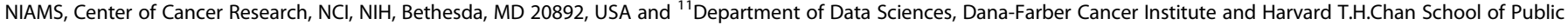
Health, Boston, MA 02215, USA

Correspondence: Fei-Long Meng (Feilong.Meng@sibcb.ac.cn)
}

These authors contributed equally: Xiaojing Liu, Tingting Liu, Yafang Shang, Pengfei Dai

Senior Authors: $X$ Shirley Liu and Fei-Long Meng

Received: 13 March 2020 Accepted: 16 April 2020

Published online: 30 April 2020 
facilitates contacts between recombining elements as well as promoters and enhancers. ${ }^{13-15}$ During CSR, chromatin loop extrusion shapes the $\mathrm{IgH}$ chromatin architecture in a spatiotemporal manner. ${ }^{15}$ Upon antigen stimulation, the activation of Ipromoters drives stepwise cohesin loading on the pre-assembled CSR center in naive B cells. ${ }^{15}$ The chromatin subdomains position the directional alignment of donor $\mathrm{S} \mu$ and acceptor $\mathrm{S}$ regions, which ensures deletional CSR in cis. ${ }^{15}$ However, the identity of the trans-acting factors behind orientation-specific end-joining remains unclear. ${ }^{16}$ DSBR factors, especially 53BP1, favor deletional CSR. ${ }^{4}$ The absence of 53BP1 is associated with excessive resection of DSB ends and near complete block of $\mathrm{CSR}_{1}{ }^{17}$ indicating that the loss of directional repair might be an indirect effect. Similarly, Lig4 deficient cells tend to have more inversional CSR joining during CSR, reflecting that the escaped broken ends from the joining complex are joined randomly in a diffusional manner at low levels. $^{18}$

To search for additional end-joining factors, we combined CRISPR knockout screening and chemical perturbation screens, and functionally characterized the hit in the context of immune diversification. The strategy identified ERCC6L2 (Excision Repair Cross-Complementation Group 6 Like 2) as a new NHEJ factor that channels programmed CSR to directional repair.

\section{RESULTS}

DNA repair genetic networks revealed by combined CRISPRchemical screens

To identify potentially new NHEJ factors, we combined chemical perturbation screens on 36 compounds with focused CRISPRknockout screens on 414 genes in the $\mathrm{CH} 12$ B cell line (Fig. 1a; Supplementary information, Table S1, see Materials and Methods for details). Genes were selected from known DNA repair factors and their homologs based on gene-ontology (GO) terms (Supplementary information, Table S1). DNA damage-inducing chemicals, including carcinogens, therapeutic agents, or DNA damage response (DDR) inhibitors, were selected based on their known functions and inhibitory concentrations (IC) in $\mathrm{CH} 12$ cells (Supplementary information, Table S1). Pools of knock-out cells were cultured with chemicals at $\mathrm{IC}_{20}$ (20\% maximal inhibitory concentration) for 12 days. Hit genes were called with the MAGeCK algorithm and presented as a z-score, ${ }^{19}$ where a negative $z$-score indicates that the knockout of a gene renders the cell more sensitive to the chemical. Clustering of the chemicals by their CRISPR screen z-scores across all the 414 genes categorize the chemicals into two main groups (Fig. 1b; Supplementary information, Fig. S1a).

The first group consists of reagents that generate single and double-strand breaks (SSBs and DSBs). The SSB subgroup included 7 poly-ADP ribose polymerase (PARP) and 4 DNA topoisomerase I (TOP1) inhibitors (Fig. 1b), consistent with the observation that these reagents produce similar DNA lesions. ${ }^{20,21}$ In the DSB subgroup, DNA topoisomerase II (TOP2) inhibitors clustered with $\gamma$-radiation mimicking reagent Zeocin, and G-quadruplexinteracting drug pyridostatin, all of which are known to induce DSBs. ${ }^{22-24}$ Although methyl methanesulfonate (MMS) does not directly cause breaks, ${ }^{25}$ the downstream lesions may be converted into DSBs at the dosage used in our screen.

The second major group contains reagents that cause blockage on DNA, such as interstrand crosslink (ICL) reagents, nucleoside analogs, crosslinkers, and DNA intercalators as well as DDR inhibitors against ATM and DNA-PKcs (Fig. 1b). This clustering suggests that blockage of DNA replication/transcription may cause the cell proliferation defects observed in the group. We conclude that the combined CRISPR-chemical screens cluster similar chemicals (illustrated by the same color block in Fig. 1b) based on the kind of DNA damage they cause, demonstrating the effectiveness of our approach at dissecting the function of DNA repair genes and DNA damage chemicals.

ERCC6L2 clusters with other NHEJ factors

Next, we clustered all 414 DNA repair genes by their z-scores across the 36 chemicals used, which categorized genes into three major groups depending on their impact on cell growth (Supplementary information, Fig. S1a). Consequently, epistatic genes segregated together, such as those involved in Fanconi anemia and NHEJ factors (Supplementary information, Fig. S1a). NHEJ factors segregated in turn into two main clusters (Fig. 1c): cluster 1 contained core subunit genes (Ku70/80, Lig4, DNA-PKCs) and potentially new members (Baz1b, Ercc6/2). Cluster 2 comprised several other known NHEJ genes: $X r c c 4, \operatorname{Paxx}, 53 b p 1$, Pol $\lambda$, Pol $\theta$ and the apurinic/apyrimidinic endonuclease gene Apex2. Mutations in $E R C C 6 L 2$ have recently been identified in inherited bone marrow failure (BMF) patients. ${ }^{26-30}$ Several classic NHEJ gene mutants have been implicated in $\mathrm{BMF}^{28}$ leading us to wonder whether ERCC6L2 contributes to NHEJ pathway.

Interestingly, ERCC6L2 deficient cells were depleted upon Zeocin treatment which induces DSBs (Fig. 1d), but not in the presence of Cisplatin or Veliparib treatment which creates ICLs and SSBs, respectively (Fig. 1e). This is consistent with results obtained from patient-derived lines carrying ERCC6L2 mutations. ${ }^{27}$ To confirm the screening results, we deleted Ercc6/2 in $\mathrm{CH} 12 \mathrm{~B}$ cells with two sets of sgRNAs. Set 1 deleted the predicted catalytic domain on ERCC6L2, while set 2 created an out of frame mutation (Supplementary information, Fig. S1b, Table S2). We found that all resulting clones were hypersensitive to treatments that induce DSBs, such as $\gamma$-irradiation (IR), Zeocin and Etoposide (Fig. 1f; Supplementary information, Fig. S1c). This phenotype is similar to, but less severe than that observed in isogenic cells lacking the major NHEJ ligase Lig4 (Fig. 1f). Increased sensitivity to DSBs was also evident in ERCC6L2-deleted Abelson virus-transformed mouse pro-B cells and human osteosarcoma U2OS cells (Supplementary information, Fig. S1d, e). Altogether, these data demonstrate that ERCC6L2 promotes DSB repair.

\section{ERCC6L2 is required for optimal CSR}

$\mathrm{V}(\mathrm{D}) \mathrm{J}$ recombination and CSR have been used to characterize the function of DSBR/NHEJ factors, and deleterious mutants of NHEJ genes frequently lead to primary immunodeficiencies (PID) (Fig. 1C). We therefore performed a focused CRISPR-knockout screen in $\mathrm{CH} 12$ $B$ cells stimulated to undergo IgM to IgA CSR, and compared the enrichment of knocked-out genes between $\operatorname{lgA}^{+}$and $\operatorname{lgM}^{+}$ populations (Fig. 2a). As controls, the screen included sgRNAs targeting known genes required for CSR (e.g. Aicda, Stat6). Consistent with previous reports, ${ }^{31}$ the assay confirmed the function of various base excision repair (BER), mismatch repair (MMR), DSBR and NHEJ factors in CSR (Fig. 2b; Supplementary information, Table S1). Among the potentially novel CSR genes, Ercc6/2 was ranked highest in the MAGeCK analysis. Conversely, other Ercc6 family members, Ercc6/CSB (functioning in transcription-coupled nucleotide excision repair) and Ercc6l/PICH (playing a role in spindle assembly checkpoint) were not required for CSR (Fig. 2b).

In cytokine activated $\mathrm{CH} 12$ cells, CSR was decreased $~ 50 \%$ in the absence of ERCC6L2, which is comparable to results in isogenic Lig4 $^{-1-}$ cells $^{32}$ (Fig. 2c). To study this phenotype under more physiological conditions, we deleted Ercc6/2 by CRISPR-Cas 9 (Fig. 2d; Supplementary information, Fig. S2a) in mouse embryos, which were either wild type or carried preassembled heavy and light chain (HL) genes. ${ }^{33,34}$ Ercc6/2 $2^{-1-}$ mice were viable (Supplementary information, Fig. S2b). Splenic naive B cells were purified from KO and control mice and stimulated with LPS or LPS + IL4 to induce CSR to lgG3 or lgG1 respectively. Consistent with the $\mathrm{CH} 12$ results, ERCC6L2 deficiency resulted in a $\sim 50 \%$ reduction in CSR (Fig. 2d; Supplementary information, Fig. S2c), with no obvious 


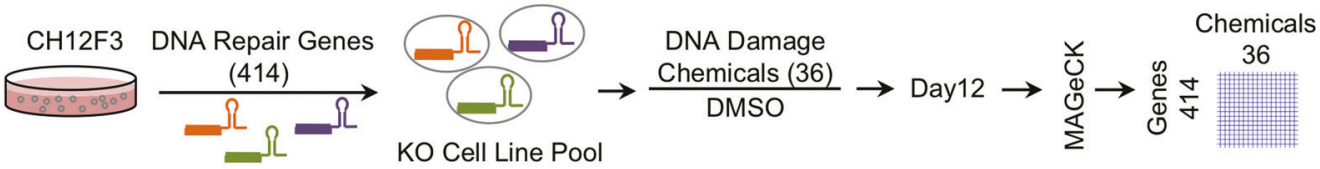

b

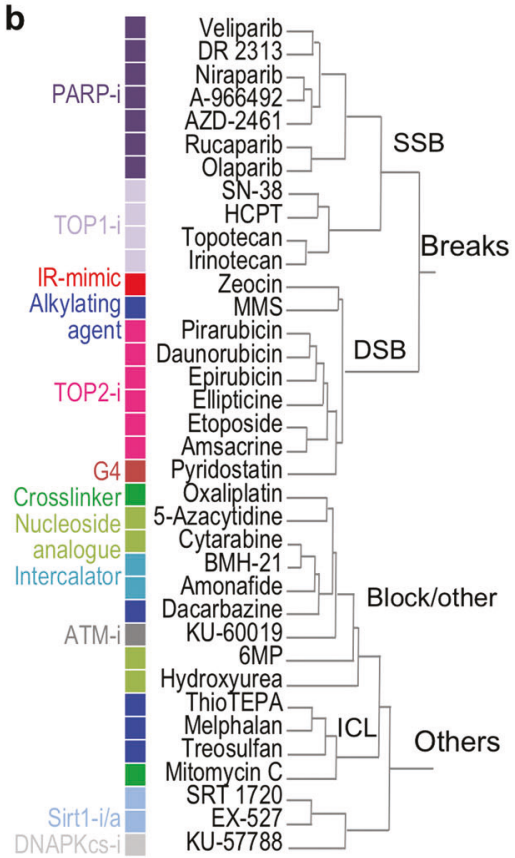

b

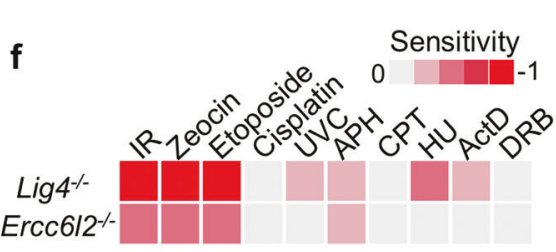

C

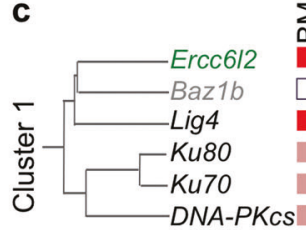

d

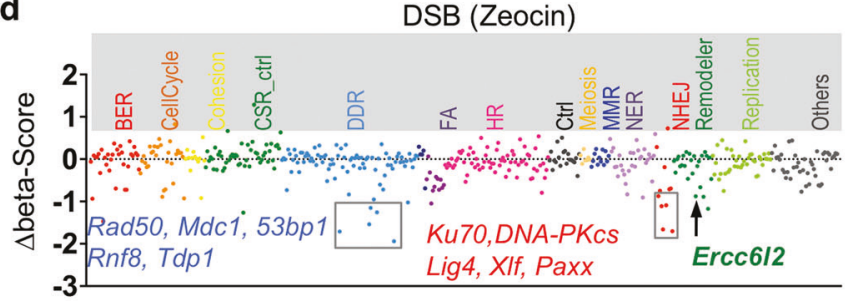

e

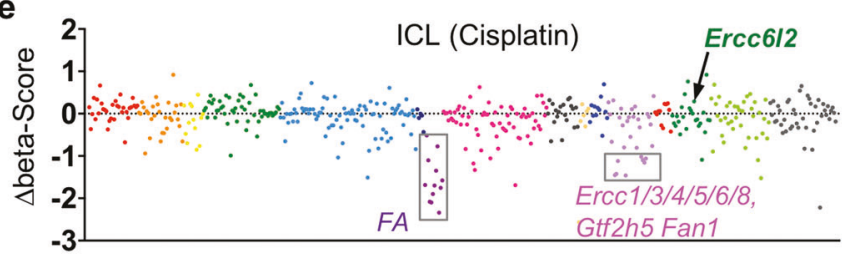

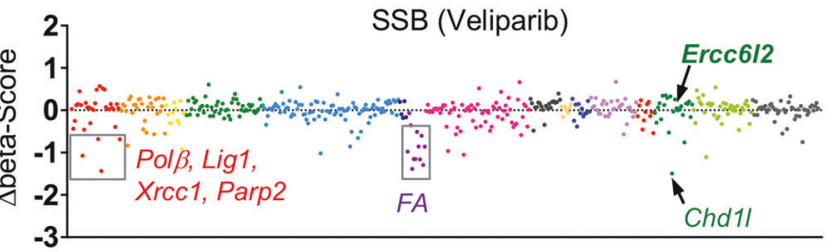

Fig. 1 CRISPR-chemical screening uncovers ERCC6L2 as a potential NHEJ factor. a Schematic illustration of chemical genetic screening procedure. b Clustering tree of chemicals. Chemical names are listed and annotated by colored blocks. Similar chemicals based on their inhibitory targets or caused DNA damages types are marked with same color. c Tree-view of two NHEJ gene clusters. Gene names are listed. Roles in bone marrow failure (BMF) or primary immunodeficiency (PID) are annotated by colored blocks. d, e Sensitivity of gene knockouts to Zeocin (d) or Cisplatin/Veliparib (e) treatment. Genes are grouped by gene ontology and depicted with different colors based on the involved biological processes along the $x$-axis. The beta-score difference (chemical-DMSO) indicating positive or negative enrichment in the chemical treatment samples was calculated from two replicates and is plotted for each gene. Representative negatively enriched genes are labeled. $F A$ indicated Fanconi anemia genes. f Sensitivity of ERCC6L2-deficient or LIG4-deficient B cells to different treatments. Cell viability curve was calculated and the area-under-the-curve (AUC) was computed. Heat map of sensitivity, which is indicated as "log ${ }_{2}$ (AUC ${ }^{\mathrm{KO}} / \mathrm{AUC}$ WT)", is plotted. IR $\gamma$-irradiation, UVC ultraviolet wavelength $254 \mathrm{~nm}$, APH aphidicolin, CPT camptothecin, HU hydroxyurea, ActD actinomycin D, DRB 5,6Dichlorobenzimidazole $1-\beta$-D-ribofuranoside.

impact in naive splenic B cell number, proliferation, AID expression, Ch gene transcription, or genome-wide transcription (Supplementary information, Fig. S2d-k, and Table S3). Correspondingly, the serum $\lg \mathrm{G} / \lg$ A levels were significantly decreased in Ercc6/2 $2^{-1-}$ mice (Supplementary information, Fig. S2I). In germinal centers of immunized mice, AID-induced somatic hypermutation (SHM) was unaffected (Supplementary information, Fig. S2m). This is reminiscent to the phenotype reported for DSBR/NHEJ-deficient germinal center $B$ cells. ${ }^{31}$ These results demonstrate that ERCC6L2 facilitates antibody isotype switching.

During CSR, AID deaminates cytosines at switch region DNA. BER and MMR enzymes convert the uracils into DSBs, which are then processed by NHEJ factors during the recombination step. ${ }^{31}$ To test whether ERCC6L2 functions upstream or downstream of DSB induction, we used Cas9 to create breaks at $\operatorname{lgH}$ switch regions in the absence of AID (Supplementary information, Table S2). Under these conditions, Cas9 can promote efficient
CSR to $\lg 1$ or $\lg A$ (a process dubbed Cas-CSR, Fig. 2e; Supplementary information, Fig. S3a). End-joining level of Cas9 breaks in the absence of ERCC6L2 was reduced to $70 \%-75 \%$ of that in isogenic control cells (Fig. 2e; Supplementary information, Fig. S3b). These results indicate that ERCC6L2 promotes general DSB end-joining. It is of note that the reduction of Cas-CSR in ERCC6L2 deficiency is less than that caused by Lig4 deficiency (Fig. 2e), while ERCC6L2 and Lig4 deficiencies had comparable effects on AID-initiated CSR (Fig. 2c). In the Cas-CSR assay, the expression of Cas9 was well controlled by the co-transfected mCherry $^{+}$control cells in the same transfection reaction (Supplementary information, Fig. S3). The levels of Cas9generated break levels among difference genotypes cannot be quantitatively revealed by the current technology. However, our observations are reminiscent of the fact that ERCC6L2 deficient cells showed less sensitivity to IR- or chemical-induced DSBs comparing to isogenic Lig4 deficient cells (Fig. 1f). Thus, it is 
a

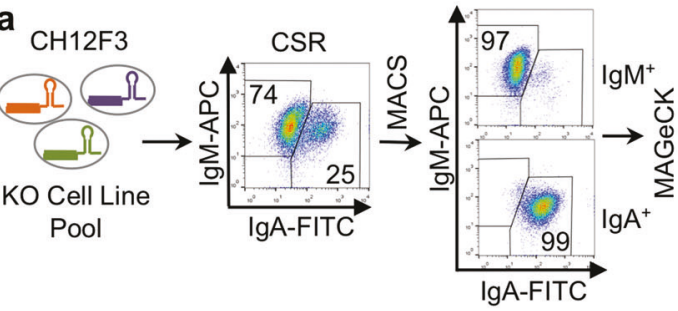

C

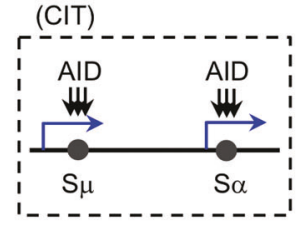

e
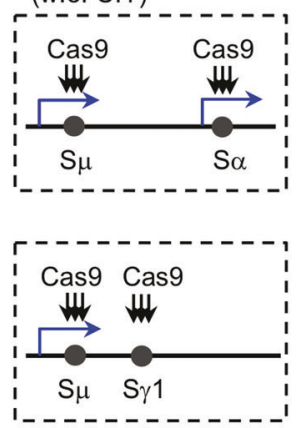

b

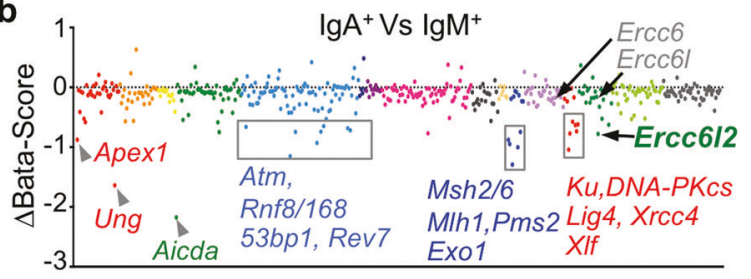

d
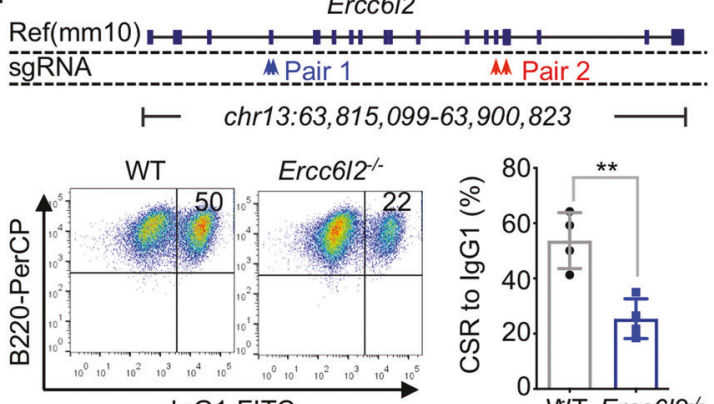

$\lg$ G1-FITC

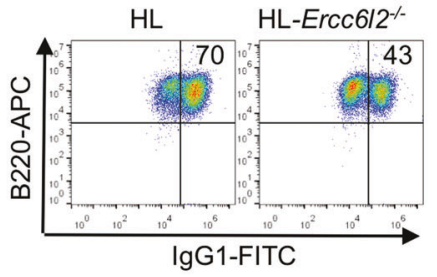

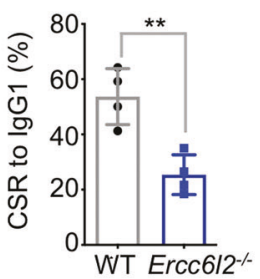

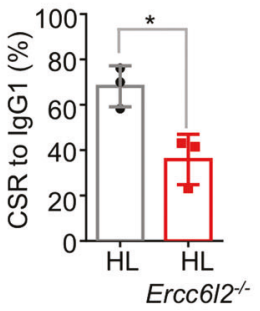

Fig. 2 ERCC6L2 is required for optimal CSR. a Schematic illustration of CSR screening procedure. Representative flow cytometry plots are

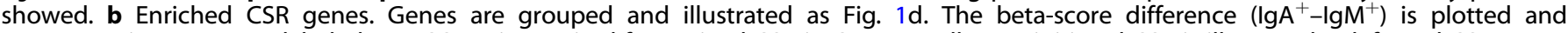
representative genes are labeled. c ERCC6L2 is required for optimal CSR in CH12F3 cells. AID-initiated CSR is illustrated at left, and CSR to IgA in presence of cytokines (CIT, $\alpha$-CD40/IL4/TGF $\beta$ ) of indicated cells are plotted at right. Blue arrows indicate transcription. Colored points indicate knockout clones obtained with different sets of sgRNAs. d ERCC6L2 is required for optimal CSR in ex vivo activated splenic B cells. Gene knockout strategies with two sets of sgRNAs are illustrated on top. Representative CSR flow cytometry plots are showed at left. Data from four pairs of Ercc6/2 ${ }^{-1-}$ (sgRNA Pair 1) and wild-type (WT) mice and three pairs of HL-Ercc6/2 ${ }^{-1-}$ (sgRNA Pair 2) and corresponding Ig heavy and light chain knockin (HL) mice are summarized. e ERCC6L2 is required for optimal Cas-CSR. CRISPR/Cas9-initiated CSR is schematically illustrated at left, and normalized CSR level of indicated cells are plotted at right. Data are represented as mean \pm SD (standard deviation) in (c, d, e). Two-tail unpaired $t$-test was performed for (c, d, e). ${ }^{* * *} p<0.0001,{ }^{* * *} p<0.001,{ }^{* *} p<0.01 ;{ }^{*} p<0.05 ; \mathbf{n s :} p>0.05$.

unlikely that ERCC6L2 affected Cas9 cutting efficiency. The comparison of AID-CSR and Cas-CSR suggests ERCC6L2 might have an additional role in physiological CSR besides end-joining.

C-terminal half of ERCC6L2 leads its catalytic activity to DNA damage sites

ERCC6L2 contains three conserved domains: a Tudor domain, an ATPase/Helicase domain and a conserved HEBO domain of unknown function (Fig. 3a). Bioinformatic analysis revealed that except for the HEBO domain, the C-terminal half of ERCC6L2 is less conserved and contains intrinsically disordered sequence by bioinformatic prediction (Supplementary information, Fig. S4a). We found that Tudor domain mutants can fully support CSR in Ercc6/2 $2^{-1-}$ cells (Fig. 3a; Supplementary information, Fig. S4b). However, ERCC6L2 N-terminal or C-terminal fragments failed to do this (Fig. 3a; Supplementary information, Fig. S4b). Furthermore, the helicase catalytic-dead (DEAH $>A A A H)$ mutant did not promote CSR (Fig. 3a; Supplementary information, Fig. S4b), indicating that ERCC6L2's predicted catalytic activity is required for DNA end-joining. Consistent with this idea, ERCC6L2 helicase catalytic-dead mutant and various frame-shift mutants (containing $\mathrm{N}$-terminal fragments only) were also identified in BMF patients (Supplementary information, Fig. S4c).

To shed further light on ERCC6L2 protein domains, we fused the full-length protein or its mutants to GFP and studies the dynamics in cells exposed to laser microirradiation. We found that GFPERCC6L2 is recruited to micro-irradiated sites within seconds of DNA damage, with similar kinetics to that of Ku70 and XLF (Fig. 3b; Supplementary information, Fig. S4d). This recruitment was observed in more than $90 \%$ of cells, and was independent of KU70, H2AX, NBS1, XLF or PARPs (Fig. 3c-f; Supplementary information, Fig. S4e). The ERCC6L2 C-terminal half fragment was sufficient to drive nuclear localization and recruitment to damaged sites (Fig. $3 \mathrm{~g}$ ), while the $\mathrm{N}$ terminal fragment could not be efficiently transported into the nucleus (Supplementary information, Fig. S4f). Remarkably, fusing ERCC6L2 ${ }^{\mathrm{N}}$ to a nuclear localization signal peptide did not rescue DNA damage foci formation (Fig. $3 \mathrm{~g}$ ). These results indicate that the C-terminal domains recruits ERCC6L2 catalytic activity to damaged chromatin.

ERCC6L2 interacts with end-joining factors

ERCC6L2 has been suggested to be an early DSB response factor. ${ }^{27}$ However, IR-induced phosphorylation of ATM substrates $\mathrm{H} 2 \mathrm{AX}, \mathrm{CHK} 2$ and KAP1 was unaffected in Ercc6/2 ${ }^{-1-}$ cells (Fig. 4a). Instead, we found that ectopically overexpressed ERCC6L2 co-immunoprecipitated with several NHEJ subunits in HEK293T cells in a DNA-independent manner (Supplementary information, Fig. S5a, b). This has been previously observed by Immunoprecipitation-Mass Spectrometry (IP-MS) analysis, ${ }^{35}$ 


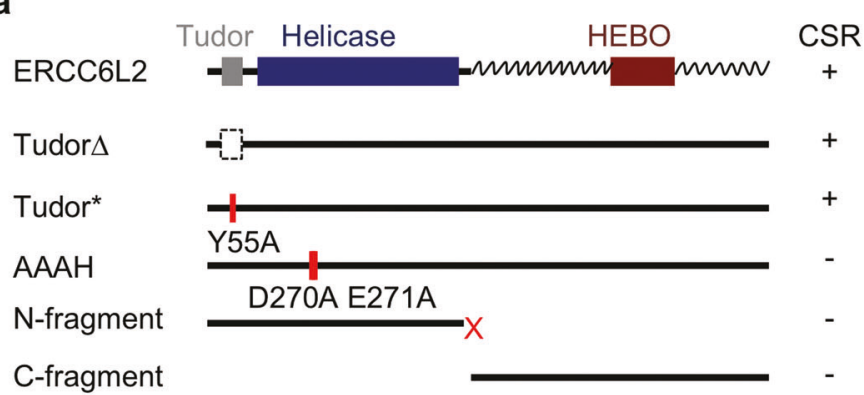

C

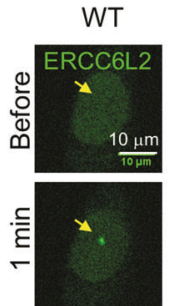

d

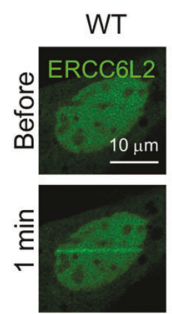

Ku70-

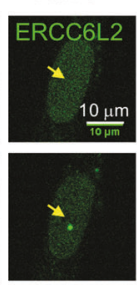

Nbs1--

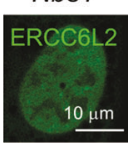

(20)
H2ax ${ }^{-1-}$

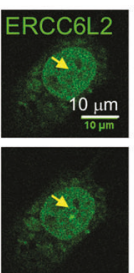

NBS1 $1^{\text {mut }} \mathbf{f}$
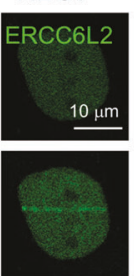

e

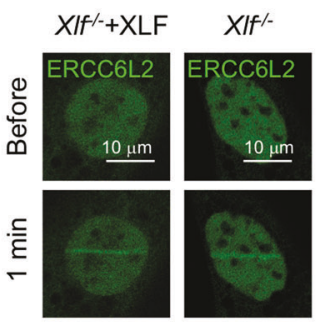

f

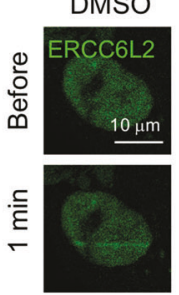

b
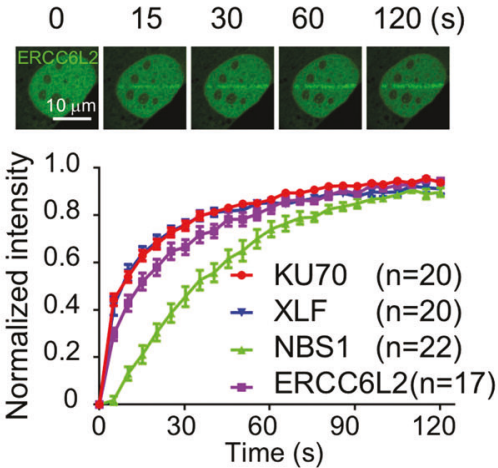

g
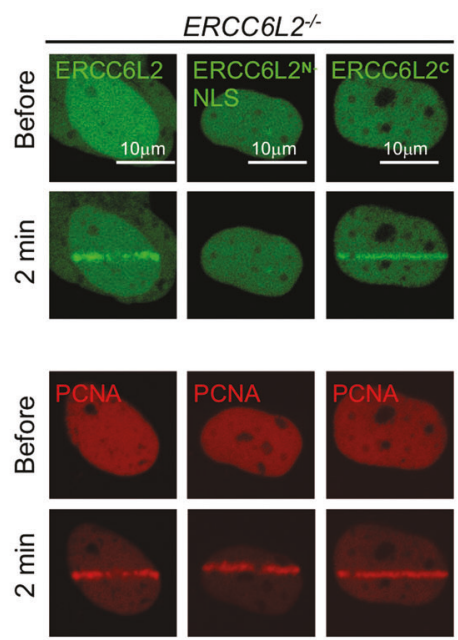

Fig. 3 ERCC6L2 quickly engages in DNA damage repair. a Summary of ERCC6L2 mutants in CSR. ERCC6L2 domain architecture is illustrated on top. The zigzag line indicates predicted internal disordered sequence. The CSR levels in ERCC6L2 mutant-complemented B cells are summarized at right. +: wild-type level, -: empty vector control level. b Recruitment of indicated proteins to microirradiation damaged sites in U2OS cells. Upper panel, a representative time-lapse view of GFP-ERCC6L2 recruitment. Lower panel, normalized fluorescent intensity of indicated proteins at DNA damage sites. The intensity was normalized to maximal intensity value at $120 \mathrm{~s}$. Data are represented as mean \pm SEM. Observed cell numbers ( $n$ ) are listed. The curves of KU70 and XLF recruitment are highly overlapped. c Representative images of ERCC6L2 recruitment in MEFs of indicated genotypes. DNA damage sites (dot) are pointed. $\mathbf{d}$ Representative images of ERCC6L2 recruitment in MEFs (left and middle) or human fibroblast (right) of indicated genotypes. e, $\mathbf{f}$ Representative images of ERCC6L2 recruitment with/without XLF (e) or in presence of $10 \mu \mathrm{M}$ PARP inhibitor Veliparib (f). $\mathbf{g}$ Representative images of ERCC6L2 fragments recruitment in ERCC6L2 ${ }^{-1-} \mathrm{U} 2 \mathrm{OS}$ cells. GFP-ERCC6L2, GFP-ERCC6L2 ${ }^{\mathrm{N}}$-NLS or GFP-ERCC6L2 $2^{\mathrm{C}}$ fusion protein was expressed in ERCC6L2 ${ }^{-1-}$ U2OS cells. The recruitment of mCherryPCNA was assayed at the same time as a control for DNA damage.

although we cannot conclude whether it is a direct or indirect interaction. Interestingly, consistent with IP-MS analysis and genome-wide yeast two-hybrid report, ${ }^{11,36}$ we observed a DNAindependent interaction between ERCC6L2 and MRI/CYREN (Fig. 4b). This interaction was mediated by ERCC6L2 Cterminus and a conserved motif in the middle of MRI/CYREN protein (Fig. 4c; Supplementary information, Fig. S5c, d).

Despite extensive testing, we were unable to obtain a workable anti-ERCC6L2 antibody or serum and currently no commercial anti-ERCC6L2 antibody is available to detect the endogenous protein. To confirm the protein interaction in vivo, we generated Ercc6/2-HA knock-in mice (Fig. 4d, top), in which the HA-tag was fused to the last exon of Ercc6/2. We detected high levels of ERCC6L2-HA protein in CSR-activated B cells (Supplementary information, Fig. S5e), where it co-immunoprecipitated with KU (Fig. 4d) and an ectopically-expressed Flag-tagged MRI (Fig. 4e). Together, these findings indicate that ERCC $6 \mathrm{~L} 2$ is associated with other NHEJ subunits.

Next, we examined how the physical interaction might contribute to ERCC6L2's function in end-joining. Similar to Ku70, MRI is not required for ERCC6L2 recruitment to DNA damage sites (Supplementary information, Fig. S6a). We then checked the recruitment kinetics of NHEJ factors in ERCC6L2 ${ }^{-1-}$ U2OS cells, and found KU70/80, PAXX and XLF to be unaffected while the recruitment of XRCC4 was slightly decreased (Supplementary information, Fig. S6b, c). To further investigate this, we generated Ercc6/2 $2^{-1-}$ MEFs. Compared to controls, there were fewer KO cells showing MRI or XRCC4/LIG4 recruitment to micro-irradiated sites, and those that did, showed a significant decrease in MRI or XRCC4/LIG4 signals at foci (Fig. 4f, g; Supplementary information, Fig. S6d). The overall expression levels of MRI, XRCC4 or LIG4 protein were unaffected by ERCC6L2 deficiency, based on total fluorescent intensity (Supplementary information, Fig. S6e). The recruitment of XLF was slightly affected in Ercc6/2 ${ }^{-/-}$MEFs (Supplementary information, Fig. S6f, $p=0.07$ ). The results indicate that the presence of ERCC6L2 at DSB sites could facilitate ligase complex loading.

ERCC6L2 is required for $\mathrm{V}(\mathrm{D}) \mathrm{J}$ recombination in sensitized XLFdeficient cells

Core NHEJ factors are absolutely required for $\mathrm{V}(\mathrm{D}) \mathrm{J}$ recombination. $^{37}$ Thus, we examined the lymphocyte development in ERCC6L2 deficient mice to check the $V(D) J$ recombination efficiency (Supplementary information, Fig. S7a). Distribution of pro-B $\left(\mathrm{CD} 43^{+} \mathrm{B}^{2} 20^{+} \mathrm{IgM}^{-}\right)$, pre-B $\left(\mathrm{CD}^{-} 3^{-} \mathrm{B}^{2} 20^{+} \mathrm{IgM}^{-}\right)$, immature $\mathrm{B}$ $\left(\operatorname{lgM}^{+} \mathrm{B} 220^{\text {low }}\right)$ and recirculating $B\left(\operatorname{lgM}^{+} B 220^{\text {hi }}\right)$ cells in Ercc6/2 ${ }^{-1-}$ 

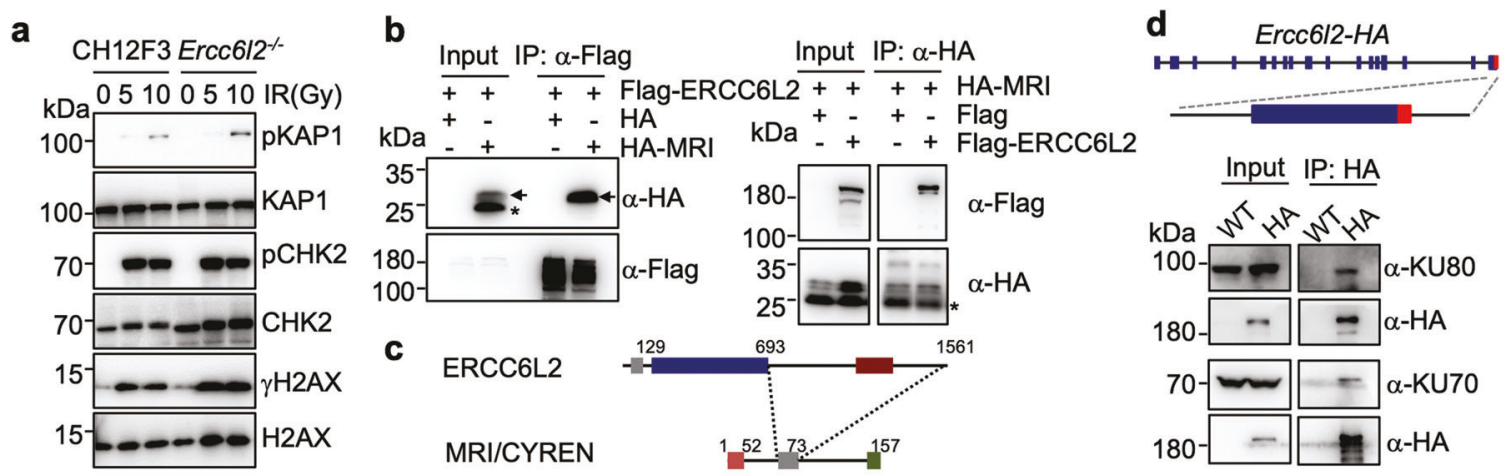

$\underline{\text { Input IP: HA }}$
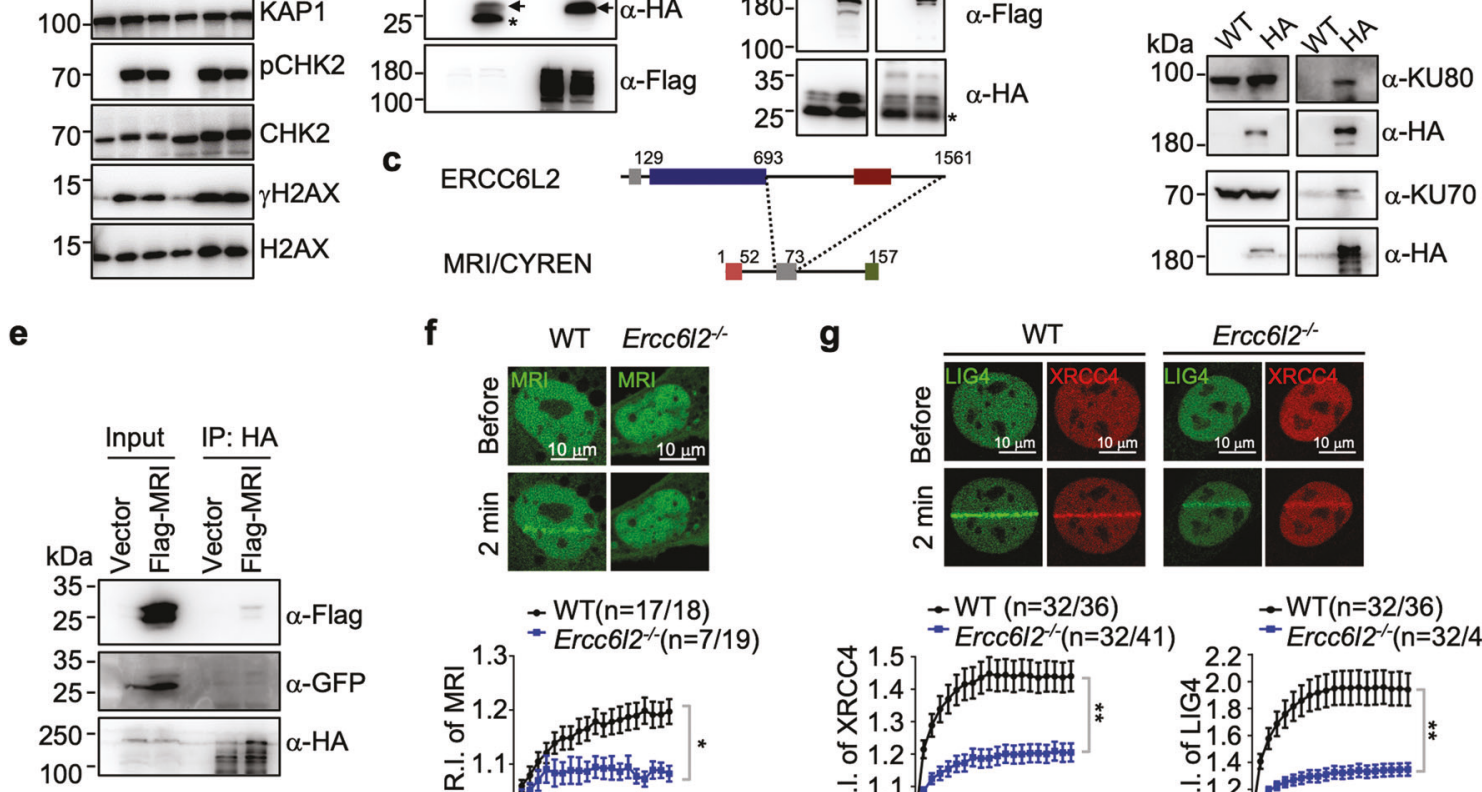
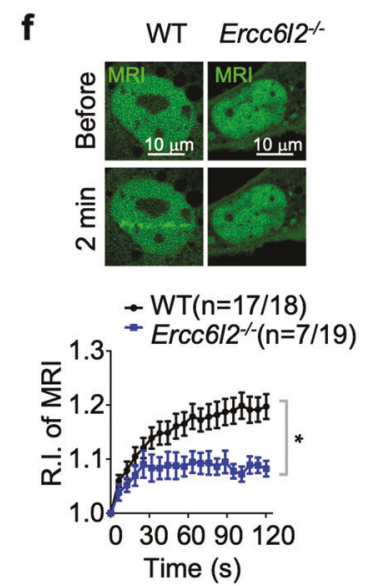

g
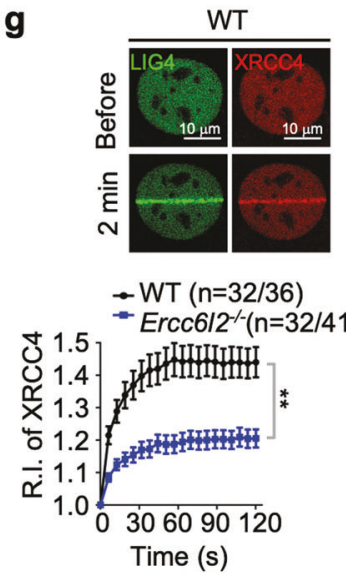
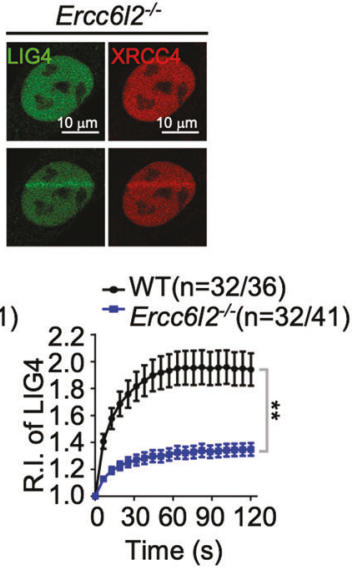

Fig. 4 ERCC6L2 is a component of end-joining machinery. a Phosphorylation of ATM substrates was examined by western blot. Indicated $\mathrm{CH} 12 \mathrm{~F} 3$ cell lines were treated with IR at different dosages, and representative blots are showed from three repeats. b Coimmunoprecipitation (Co-IP) of ERCC6L2 and MRI/CYREN in HEK293T cells. The panels show western blots with anti-Flag or anti-HA antibodies of total extract (input) and IP samples. Arrows indicate bands of HA-MRI. Asterisk marks an unknown form of HA-MRI that does not interact with ERCC6L2. c Schematic illustration of ERCC6L2-MRI interaction domains. d Inputs and anti-HA-immunoprecipitated samples from CSR-activated B cells were assayed for NHEJ subunits. WT: B cells from wild-type mice; HA: B cells from Ercc6/2-HA knock-in mice. Knock-in strategy of Ercc6/2-HA mice is showed at top. e Inputs and anti-HA-immunoprecipitated samples from Ercc6/2-HA knock-in CSR-activated B cells with retroviral-expressed Flag-tagged MRI. Flag-MRI-IRES-GFP was ectopically expressed in CSR-activated B cells with an empty retroviral vector (IRES-GFP) as control. IRES internal ribosome entry site. f Recruitment of MRI to DNA damage site in WT and ERCC6L2-deficient MEFs. Upper, total numbers of micro-irradiated cells are listed alone with numbers of cells showed detectable MRI accumulation. Percentage of cells with MRI accumulation are showed in parenthesis. Exampled images of GFP-MRI recruitment are showed at lower. Relative intensity (R.I.) of GFP-MRI at DNA damages are calculated. g Recruitment of XRCC4/LIG4 to DNA damage site in WT and ERCC6L2-deficient MEFs. GFP-LIG4 and mCherry-XRCC4 were co-expressed in cells, and the panel is illustrated as in (f). Data are represented as mean \pm SEM in (f) and (g). A $t$-test was applied as described in the "Materials and Methods" section. ${ }^{* *} p<0.01$; ${ }^{*} p<0.05$.

bone marrow was similar to that of WT. Although the total cell numbers in the $\operatorname{Ercc} 6 / 2^{-1-}$ thymus were $~ 50 \%$ of that in WT, the distribution of $\mathrm{CD}^{-} \mathrm{CD}^{-}, \mathrm{CD}^{+}{ }^{+} \mathrm{CD} 8^{+}, \mathrm{CD}^{+}{ }^{+} \mathrm{CD} 8^{-}$and $\mathrm{CD} 4^{-} \mathrm{CD}^{+}$ Ercc6/2 $2^{-1-}$ T cells were comparable to that of WT (Supplementary information, Fig. S7a). The lymphocyte development in ERCC6L2 deficient mice is different from that in core NHEJ factor deficiency. ${ }^{37}$ Previous studies have uncovered a functional redundancy between XLF and ATM-dependent DSB response factors, or between XLF and other non-essential NHEJ factors including PAXX and MRI. ${ }^{1,38-44}$ Therefore, we hypothesized that ERCC6L2 may play a role in $V(D) J$ recombination that is masked by other NHEJ factors, possibly XLF. To test this idea, we performed a focused CRISPR screen in WT and XIf-l- $v$-Abl lines with a modified pMX-INV substrate (Fig. 5a; Supplementary information, Fig. S7b, c). DNA repair genes required for recombination were identified as negatively enriched in the recombined $\mathrm{hCD}^{+}$population (see Materials and Methods). As expected, core NHEJ factors and Artemis were identified in both genotypes (Fig. 5b). Also, as previously reported, ${ }^{39-44}$ XLF was functionally redundant with PAXX, 53BP1, and H2AX. The assay however identified new XLF redundant factors, including NBS1, MDC1, RNF8, RNF168, INO80, and importantly ERCC6L2 (Fig. 5b). Of note, Mri was not included in our original focused CRISPR sgRNA library (Supplementary information, Table S1), so Mri did not show up here.

The functional redundancy between ERCC6L2 and XLF was confirmed by chromosomal $V(D) J$ recombination assays in Ercc6l2 $2^{-1-} \mathrm{XIF}^{-1-}$ and isogenic $\mathrm{v}-\mathrm{Abl}$ lines (Fig. 5c; Supplementary information, Fig. S7d). At the same time, there was no obvious redundancy in Ercc6/2 ${ }^{-1-} \mathrm{Paxx}^{-1-}$ cells. Similar to Lig4 $^{-1-}$, Ercc6/2 ${ }^{-1-}$ XIf $^{-1-}$ cells showed a near complete block in V(D)J recombination (Fig. 5c; Supplementary information, Fig. S7d). This defect could be rescued by ectopic expression of ERCC6L2 but not ERCC6L2 N-terminal fragment (Supplementary information, Fig. S7e). Absence of coding joins and a smear signal of coding ends were also noticed in Ercc6/2 ${ }^{-1-}$ XIf $^{-1-}$ cells (Fig. $5 \mathrm{~d}$ ), reminiscent of extensive resection of unprocessed DNA ends. In summary, ERCC6L2 and XLF play functional-redundant roles in the repair of DNA ends during $V(D) J$ recombination.

ERCC6L2 controls the orientation of DNA recombination

Our experiments have so far conclusively established ERCC6L2 promoting DSB end-joining in conjunction with $\mathrm{XLF}$, primarily during programmed recombination in lymphocytes. The precise molecular mechanism however is unclear. We thus applied high- 
a

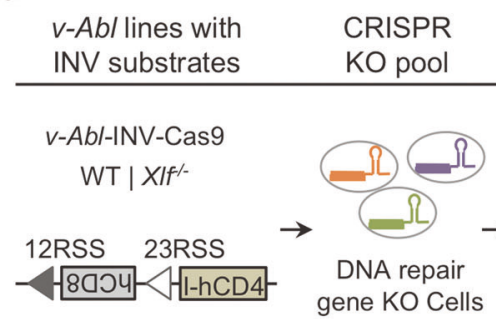

c

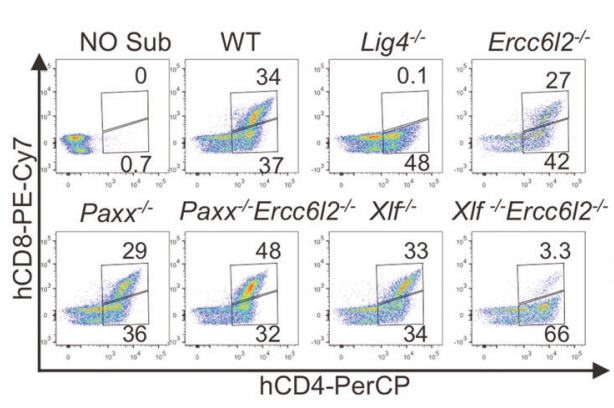

b

\begin{tabular}{llll}
\multicolumn{4}{c}{ Degative enriched genes } \\
\hline \multicolumn{1}{c}{ WT } & \multicolumn{3}{c}{ XIf $^{-2}$} \\
\hline Lig4 & Lig4 & Paxx & \\
Artemis & Artemis & & \\
Ku70/80 & Ku70/80 & & \\
DNA-PKcs & DNA-PKcs & Nbs1 & Rnf8 \\
Xrcc4 & Xrcc4 & H2ax & Rnf168 \\
XIf & & Mdc1 & $53 b p 1$ \\
Atm & Atm & Ino80 & Ercc6/2 \\
Setx & Setx & Inot
\end{tabular}

Gene category: NHEJ; DSBR; others. 

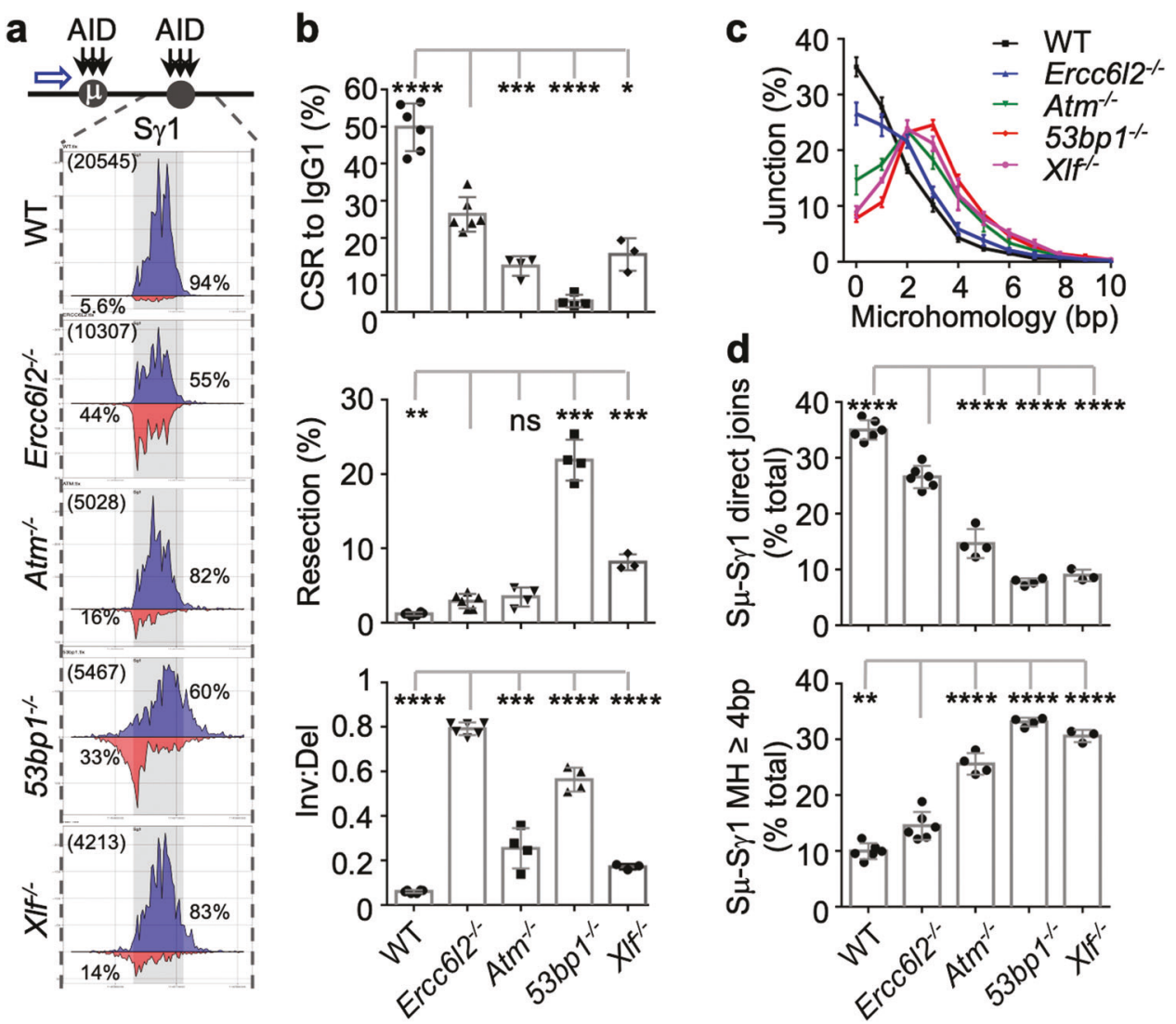

Fig. 6 ERCC6L2 determines orientation-specific recombination. a Distribution of S junctions in CSR-activated B cells. Top, AID targeting (black arrow) in $S_{\mu}$ and $S_{\gamma}$, and HTGTS bait site (blue arrow) are depicted. Bottom, linear distribution of pooled junctions along $\mathrm{S}_{\gamma} 1$ and flanking regions recovered from LPS/IL4-stimulated B cells. Gray boxes indicate $S_{\gamma} 1$. Blue, junctions orientated from left to right; Red, Junctions orientated from right to left. Total $S_{\mu}-S_{\gamma} 1$ junction number (bracketed) and percentages of inversional/deletional junctions in the region are labeled. b CSR to IgG1, percentage of resection, and inversional/deletional joining ratio are plotted from top to bottom for the indicated $B$ cells. Data were from 3 to 6 mice. c Microhomology usage of $S_{\mu}-S_{\gamma} 1$ DNA rearrangement junctions in the indicated activated $B$ cells. Percentage of junctions with different length of microhomology are plotted. d Percentages of direct joins of $5^{\prime} S_{\mu}$ to $S_{\gamma} 1$ (upper panel) and percentage of junctions with $4 \mathrm{bp}$ or longer $\mathrm{MH}$ in $\mathrm{S}_{\gamma} 1$ (lower panel) in activated B cells with indicated genotypes. Data are represented as mean \pm SD in (b, c, d). Two-tail unpaired $t$-test was performed for (b) and (d). Data from Ercc6/2 knockout are compared with those from other genotypes. ${ }^{* * *} p<0.0001,{ }^{* *} p<0.001,{ }^{* *} p<0.01 ;{ }^{*} p<0.05$, ns: $p>0.05$.

\section{DISCUSSION}

CRISPR-chemical screens dissect the function of DNA repair factors In this study, we used a comprehensive CRISPR and chemical screening approach to define a core DNA repair genetic network. In our $414 \times 36$ screen matrix, functionally-related genes or chemicals clustered together, offering new insights into gene function and how DNA damage-causing agents impact cells. Even though our focused CRISPR screening could not cover the whole genome, it has the advantage of better signal-to-noise ratios which can easily pinpoint regulators involved in subtle mechanisms. Our focused approach successfully identified factors that impact recombination by $\sim 50 \%$. Focused CRISPR screens can also be used as a reverse genetic tool to dissect genetic interactions, as we demonstrated with $\mathrm{V}(\mathrm{D}) \mathrm{J}$ recombination. Although our studies focused on the role of ERCC6L2 in NHEJ, it is worth noting that the assay revealed new functions of many other factors, including $B A Z 1 B$, a bromodomain-containing protein involved in $\mathrm{H} 2 \mathrm{AX}$ phosphorylation. ${ }^{45}$ Moreover, our data provided important hints on combinatorial cancer treatments, since many of the DNA damage reagents tested are widely used in the clinic.

A working model of ERCC6L2 function

During immune diversification, programmed DNA breaks are processed into productive rearrangements within constrained topological microenvironments or TADs. We have shown that ERCC6L2 regulates how DNA ends are joined in a spatially oriented manner during repair (Fig. 7c). During CSR, transcribed S regions are synapsed and recruit AID deamination activity, which in turn engages BER and MMR enzymes that convert uracils into staggered DNA breaks with G-rich repetitive sequences. ${ }^{3}$ Several chromatin features appear to facilitate this activity, including Rloops, ${ }^{46}$ nascent G-rich RNA, ${ }^{47}$ and paused transcription complexes. $^{48}$ These features, along with tightly packed nucleosomes, might slow down or interfere with end-joining.

In this context, ERCC6 family members are believed to function as nucleosome remodelers or DNA translocase. ${ }^{49-51}$ It is therefore tantalizing to speculate that ERCC6L2 removes nucleosomes or other protein/nucleotides near DNA lesions, probably to facilitate XRCC4/Lig4 sliding towards DNA ends. ${ }^{52}$ This function might ensure rapid in situ ligation of DSBs in the pre-assembled chromatin subdomains. Another non-mutually exclusive model is that AID-generated stagger ends within $S$ regions could be misaligned and ERCC6L2 activity could detach the mis-aligned ends, leading to the dissociation of the intervening sequence from the CSR centre. ${ }^{15}$ Thus, ERCC6L2 could be directly involved in the spatiotemporal formation of $\mathrm{lgH}$ chromatin architecture, ${ }^{15}$ which is of great interest for further investigation. These possibilities suggest a model (Fig. 7c) on how ERCC6L2 facilitates deletional 
a
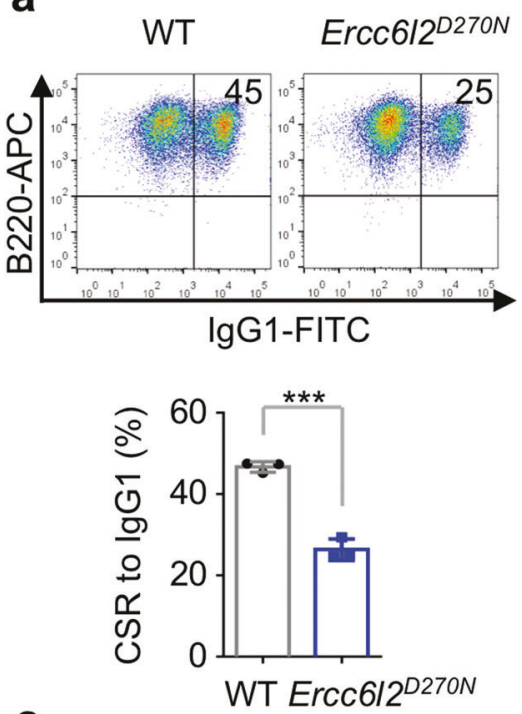

b
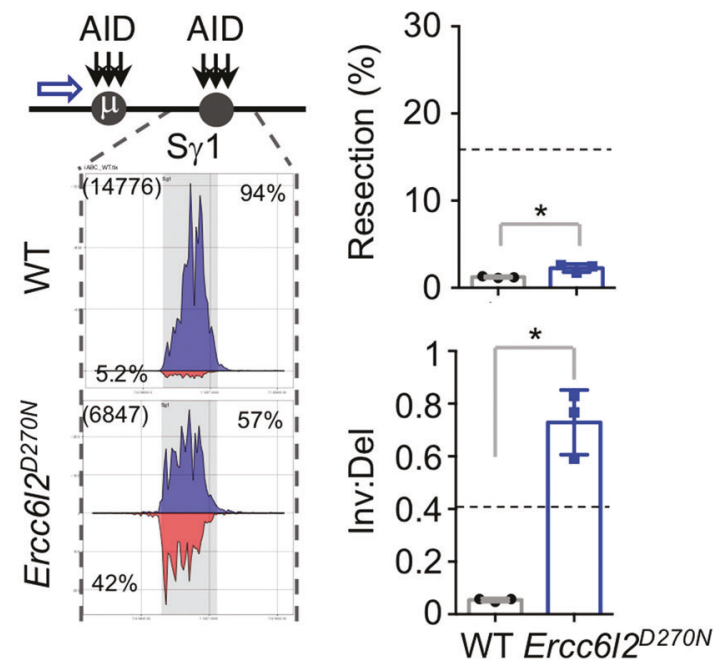

C
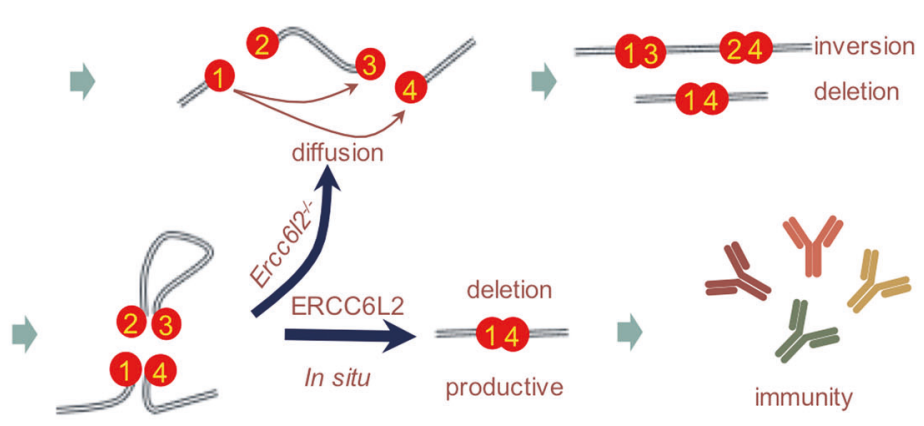

Programmed

DNA recombination
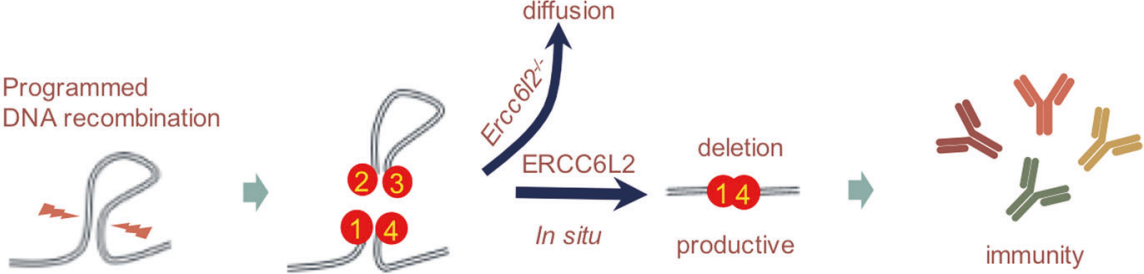

Fig. 7 ERCC6L2 activity is required for directional end-joining during CSR. a CSR levels to lgG1 in CSR-activated WT and Ercc6/2 ${ }^{D 270 N}$ B cells at Day 4 upon LPS/IL4 stimulation. b Distribution of $S_{\mu}-S_{\gamma} 1$ junctions at $S_{\gamma}$, end-resection and inversion/deletion ratio of CSR junctions in WT and Ercc6/2 ${ }^{D 270 N} \mathrm{~B}$ cells. Dashed lines indicate the levels in $53 b p 1^{-1-} \mathrm{B}$ cells, which were assayed at the same time. c A working model to explain roles of ERCC6L2, see "Discussion" for details. Data are represented as mean \pm SD and two-tail unpaired $t$-test was performed in (a, $\mathbf{b}$ ). ${ }^{* * *} p<0.001,{ }^{*} p<0.05$.

CSR of antibody genes. Considering that ERCC6L2 is the latest evolved member of the ERCC6 family, we speculate that it might have evolved to promote efficient end-joining under more complicated and specialized settings, such as in lymphocytes of higher organisms.

DNA damage in ERCC6L2-linked pathologies Mutations of ERCC6L2 and LIG4 were previously identified in BMF patients, where DNA damage may originate from the same source. In Fanconi anemia, a subtype of BMF, ICLs generated by reactive aldehydes appear to be the major source of DNA damage in HSCs and other progenitor cells. ${ }^{53}$ However, the DNA lesions underlying ERCC6L2- and NHEJ-pathologies might be of different origin, as cells isolated from such patients are typically not hyper-sensitive to ICL-inducing agents. ${ }^{27}$ Our DNA repair genetic screen provided some hints in this regard, in which it showed that NHEJ and ERCC6L2 knockouts are markedly sensitive to TOP2 inhibitors. TOP2B-induced lesions are known to accumulate genome-wide at chromosome loop anchors, ${ }^{14,54}$ and at promoters of key genes during neuronal stimulation. ${ }^{55}$ Notably, some ERCC6L2-mutated BMF patients also show neurological dysfunctions. ${ }^{26,27,30}$ Whether TOP2B-induced breaks are causal to ERCC6L2- and NHEJ-pathologies represents an interesting future line of research.

\section{MATERIALS AND METHODS}

Mice

Ercc6/2 and XIf knockout mouse lines were constructed through zygote injection of CRISPR/Cas9 constructs. Ercc6/2-HA knock-in mouse line was constructed by oocyte injection of androgenetic haploid embryonic stem cells harboring an Ercc6/2-HA allele obtained with homologous recombination (HR). Ercc6/2 $270 \mathrm{~N}$ mouse line was constructed by zygote injection of CRISPR/Cas9 constructs and a single-strand HR template. Guide RNA sequences are listed in Supplementary information, Table S2. 53bp $1^{-1-56}$ and $\mathrm{Atm}^{-1-57}$ mouse lines have been described previously. All animal experiments were performed under protocols approved by the Institutional Animal Care and Use Committee of Shanghai Institute of Biochemistry and Cell Biology.

Cell lines

Cell lines used in this study were listed in the Supplementary information, Table S2. Parental B-lineaged $\mathrm{CH}_{12} \mathrm{~F} 3$ cell line and isogenic Lig $^{-1-32}$ cell line, parental Abelson virus-transformed $(v-A b l)$ mouse pro-B cell line containing the $E \mu-B c l 2$ transgene ${ }^{43}$ have been described previously. $\mathrm{CH} 12 \mathrm{~F} 3$ and its derived isogenic cells were cultured with RPIM1640 (10-040-CV; Corning), $\beta$ Mercaptoethanol (M6250-500ML; Sigma-Aldrich), PenicillinStreptomycin-Glutamine (10378016; Thermo Fisher Scientific), 
plus 10\% FBS (FCS500; ExCell Bio), and $v$-Abl cells were cultured with RPIM1640 (10-040-CV, Corning), $\beta$-Mercaptoethanol (M6250500ML; Sigma-Aldrich), Penicillin-Streptomycin-Glutamine (10378016; Thermo Fisher Scientific), Sodium Pyruvate (11360670; Thermo Fisher Scientific), MEM Non-Essential Amino Acids Solution (11140050; Thermo Fisher Scientific), HEPES(15630080; Thermo Fisher Scientific), plus 10\% FBS (35-053-CM; Corning). MEF, HEK293T and U2OS cells were cultured with DMEM (10-013-CV, Corning), PenicillinStreptomycin-Glutamine (10378016; Thermo Fisher Scientific), plus 10\% FBS (FSP500, ExCell Bio).

All cell lines are negative for mycoplasma contamination.

Primers, plasmids and antibodies

Primers, plasmids and antibodies used in this study were listed in the Supplementary information, Table S2.

Focused CRISPR screenings and data analysis

DNA repair-related CRISPR library design. DNA repair genes were picked based on gene ontology within AmiGO 2 database (http://amigo.geneontology.org/amigo). We also chose several CSR related genes from literature serving as potential CSR positive controls, and genes affecting cell viability (essential genes) and non-essential genes serving as CRISPR screening controls. Genes are listed in Supplementary information, Table S1. Guide RNAs were designed with CRISPR-FOCUS. ${ }^{58}$ Oligo pool was synthesized by Synbio Technologies (Suzhou) and cloned into lentiGuide-Puro by using EasyGeno Assembly kit (VI201; TIANGEN). Pooled sgRNA viruses were used to infect $\mathrm{CH} 12 \mathrm{~F} 3$ or $v-A b l$ cell lines stably-expressing Cas 9 protein. Resulting cell pools were selected with Puromycin for 7 days (Day -6 to Day 0) allowing efficient KO of targeted genes, before subjected to chemical treatment, CSR or V(D)J recombination assay.

Chemical treatment in $\mathrm{CH} 12 \mathrm{~F} 3$ cells. Inhibitory concentration (IC) was determined for each chemical in B-lineaged $\mathrm{CH}_{12} \mathrm{~F} 3$ cells (Supplementary information, Table S1). Gene knockout cells were cultured with DNA damage chemicals at $\mathrm{IC}_{20}$ for 12 days (from Day 0 to Day 12) before harvested for genomic DNA purification. DMSO at a concentration of $0.1 \%$ was included as a control.

Enrichment of B cell sub-populations. For CSR screening, $\lg \mathrm{A}^{+}$ cells were labeled with Biotin-conjugated anti-lgA antibody (407003; Biolegend), which were further enriched with antibiotin microbeads (130-090-485; Miltenyi). Three replicates were performed for CSR screening of each genotype. For $V(D) J$ recombination screening, $v-A b l$ cells were stimulated by STI-571 for 4 days, $\mathrm{hCD}^{+} \mathrm{hCD}^{+}$and $\mathrm{hCD}^{-} \mathrm{hCD}^{+}$populations were enriched with anti-hCD4 (130-045-101; Miltenyi) and anti-hCD8 microbeads (130-045-201; Miltenyi). Two repeats were performed for $V(D) J$ recombination screening of each genotype.

Library preparation and data analysis. The sgRNA sequences were PCR-amplified according to previous reported method, ${ }^{59}$ and subjected to Illumina sequencing. The raw reads were trimmed with fastx_trimmer in FASTX-Toolkit, and sgRNA sequences were counted by MAGeCK. ${ }^{19}$ Clonal batch difference was controlled by using a batch-removal sub-module in MAGeCK-Flute, and enriched genes were retrieved with MAGeCK test subcommand.

Cluster of chemical treatment result. We performed hierarchical clustering for both drugs and genes by $\mathrm{R}$ programming language. First, we used dist function to compute Euclidean distances for drugs or genes by using z-score computed by MAGeCK-MLE submodule. Then the distance matrix was passed to hclust function for clustering using ward.D method.
Exclude cell fitness genes and identify CSR/V(D)J recombination factors. We found that cell fitness genes which affect cell viability or growth rate often were retrieved in different CRISPR screening assays as false positive hits. Thus, we identified the cell fitness genes in DNA repair gene list by comparing recovered sgRNAs at day 7 after puromycin selection and sgRNAs in viral vector. The list was named as "Genes_Affecting_Cellular_Fitness" in Supplementary information, Table S1. After retrieving the enriched genes from CSR or $V(D)$ J recombination screenings, cell fitness genes were first removed from the result. A false discovery rate (FDR) < 0.2 was further applied.

Chemical sensitivity assay

Cells were plated at a concentration of $6 \times 10 \mathrm{E} 4$ cells $/ \mathrm{ml}$ with indicated chemicals or treated with indicated doses of X-ray or UV. After $48 \mathrm{~h}$, cell viability was measured with a Cell Counting Kit-8 assay (K1018; APExBio). The survival data were fit to a mixedeffects model using Imer function from the R package 'Ime4', with "dosage" and "genotype" as fixed-effects parameters, and "cell viability data from each repeat" as the random effect parameter. The significance of the fixed effects parameters is obtained by the $t$-test.

Antibody class switch recombination assays

AID-initiated CSR assay. $\mathrm{CH} 12 \mathrm{~F} 3$ cells were stimulated with a-CD40 (16-0402-86; eBioscience), IL4 (CK15; Novoprotein) plus TGF- $\beta$ (CA59; Novoprotein), and CSR to IgA were monitored at day 2 and 3. Splenic naive $B$ cells were purified and cultured as previous described. ${ }^{60}$ CSR to $\operatorname{lgG} 1$ or $\operatorname{lgG} 3$ was monitored at day 3 and 4.

Cas9-initiated CSR assay. For CRISPR/Cas9-initiated CSR (Cas-CSR) in $\mathrm{CH} 12 \mathrm{~F} 3$ cells, test cells were mixed with control $\mathrm{CH} 12 \mathrm{~F} 3$ mCherry cells at a ratio of 3:1, sgRNAs targeting up- and downstream $\mathrm{S}$ regions were transfected into the mixture via electroporation. The $\mathrm{CH} 12 \mathrm{~F} 3-\mathrm{mCherry}$ controls for electroporation decreased technical variations among each transfection. CSR level to other $\mathrm{lg}$ in $\mathrm{KO}$ cells was first normalized to the CSR level of $\mathrm{CH} 12 \mathrm{~F} 3-\mathrm{mCherry}$ cells which was transfected in the same cuvette, and the relative CSR level was defined as a ratio of the CSR levels in $\mathrm{KO}$ and parental $\mathrm{CH} 12 \mathrm{~F} 3$ cells as below:

$K O$ CSR level at transfection \#1:

$\mathrm{a}=\left(\mathrm{KO}^{\operatorname{lgG}+} / \mathrm{KO}^{\mathrm{All}}\right) /\left(\mathrm{mCherry} \# 1^{\mathrm{lgG}+} / \mathrm{mCherry}^{\mathrm{All}} 1^{\mathrm{Al}}\right)$

WT CSR level at transfection \#2:

$\mathrm{b}=\left(\mathrm{WT}^{\mathrm{lgG}+} / \mathrm{WT}^{\mathrm{All}}\right) /\left(\right.$ (mCherry\#2 $\left.{ }^{\mathrm{lgG}+} / \mathrm{mCherry}^{\mathrm{All}}\right)$

Relative Cas-CSR level $=a / b$.

It is of note that in the Cas-CSR assay, the Cas9-generated break levels among difference genotypes cannot be quantitatively revealed by the current technology. Thus, decreased Cas-CSR level could also be resulted from the low Cas 9 cutting efficiency in a specific genotype.

Somatic hypermutation assay

Peyer's Patch GC B cells (B220 ${ }^{+}$PNA $^{\text {hi }}$ ) were sorted from indicated mice. $J_{H} 4$ and $J_{K} 5$ introns were PCR amplified as previously reported ${ }^{61,62}$ and the PCR products were further tagged with illumine P5 and P7 index primers and subjected to Illumina HiSeq. Data were analyzed as previously described. ${ }^{63}$

Chromosomal $\mathrm{V}(\mathrm{D}) \mathrm{J}$ recombination assay

The $v$ - $A b /$ cells were infected with INV-invert-hCD8a cassette and enriched by using anti-hCD4 microbeads (130-045-101; Miltenyi). Cells which already underwent $V(D) J$ recombination were removed with hCD8 microbeads (130-045-201; Miltenyi) at each experiment. Cells were stimulated with STI-571 for $48 \mathrm{~h}$ and $96 \mathrm{~h}$, and then subjected to flow cytometry and/or southern blot. Southern blot was performed as previously described. ${ }^{39}$ For each sample, $10 \mu \mathrm{g}$ genomic DNA was digested by Nde I/Nhe I and Nco 
I/Nhe I, respectively. A $900 \mathrm{bp}$ Hind III/Nhe I fragment of hCD4 sequence was used as the probe.

Lymphocyte development

Flow cytometry was applied to assay lymphocyte development. Bone marrow and lymphocyte cellularity were calculated by using a hemocytometer. In brief, bone marrow cells were isolated by flushing or by crushing the long bones with a mortar and pestle in $\mathrm{Ca}^{2+}$ and $\mathrm{Mg}^{2+}$ free HBSS with $2 \%$ heat-inactivated serum. Spleen and thymus cells were obtained by crushing the tissue between two glass slides. The cells were filtered through a $70 \mu \mathrm{m}$ nylon mesh before staining.

Gene deletion and complementation in cell line

For gene deletion in cell lines, pairs of sgRNAs were designed with SSC programe ${ }^{64}$ and selected based on the published gene knockout strategy or gene function domains. A GFP-expressing plasmid and pX330-based CRISPR/Cas9 plasmids were COtransfected into cells. Twenty-four hours after transfection, GFPhigh cells were sorted with BD FCAS Aria III and plated into single clones in 96-well plates. Individual clones were genotyped by PCR (Supplementary information, Table S2) and positive clones were further confirmed by western blot or RT-qPCR. Despite many attempting, we were unable to get an anti-ERCC6L2 antibody or serum to detect the endogenous ERCC6L2 protein. Thus, the knockout of Ercc6/2 was confirmed by PCR-genotyping and RNASeq. ERCC6L2 plasmids were constructed by PCR with total CDNA templates from either mouse activated-B cells or human HEK293T cells. The gene was cloned into a lentiviral vector. The resulting lentiviral particles were used to generate Ercc6/2 or Ercc6/2-mutant cell lines. Empty vector (EV) was used as control. The Ercc6/2 mutant genes express at similar level as the WT gene in the experimental conditions.

Laser micro-irradiation

Laser microirradiation was performed as described. ${ }^{39}$ Briefly, U2OS cells were plated on $35 \mathrm{~mm}$ diameter glass-bottom plates (D35-20$1-\mathrm{N}$, Cellvis) at $1 \times 10 \mathrm{E} 5$ cells $/ \mathrm{ml}$. On the next day, $1 \mu \mathrm{g}$ GFP-GENE fusion plasmid with $0.05 \mu \mathrm{g}$ mCherry-PCNA plasmid were transfected into cells. For MEFs, $10 \mu \mathrm{g}$ GFP-GENE fusion plasmid was transfected into cells by electroporation (VCA-1001, Lonza Nucleofector 2b) following the manufacture's instruction. The next day after transfection, cells were incubated with $10 \mu \mathrm{M} 5$ BrdU (HY-15910; MedChemExpress) overnight and exposed to 10 $\mu \mathrm{g} / \mathrm{ml}$ Hoechst 33342 (C1022; Beyotime Technology) for $10 \mathrm{~min}$ just before irradiation. Laser microirradiation was performed with Nikon A1 confocal microscope and a $405 \mathrm{~nm}$ laser with $100 \%$ energy. The fluorescence data were fit to a mixed-effects model using Imer function from the R package 'Ime4', with "acquired time" and "genotype" as fixed-effects parameters, and "data from each observed cell" as the random effect parameter. The significance of the fixed effects parameters is obtained by the $t$ test.

\section{Immunoprecipitation}

Co-transfection of GFP tagged NHEJ subunits and 3xFlag-ERCC6L2 or HA-ERCC6L2 in HEK293T cells was performed using Lipofectamine 2000 according to the manufacturer's instruction. After $48 \mathrm{~h}$, cells were washed, scraped and lysed for $30 \mathrm{~min}$ in lysis buffer (10 $\mathrm{mM}$ Tris- $\mathrm{HCl} \mathrm{pH} 7.4,150 \mathrm{mM} \mathrm{NaCl}, 2.5 \mathrm{mM} \mathrm{MgCl} 2,10 \%$ Glycerol, $0.5 \%$ NP-40), supplemented with Complete protease inhibitor mix (4693132001, Roche), 25 U/ml Benzonase (E8263, Sigma-Aldrich) and $20 \mu \mathrm{g} / \mathrm{ml}$ Ethidium Bromide. Lysates were centrifuged at $16,000 \mathrm{~g}$ for $10 \mathrm{~min}$ at $4{ }^{\circ} \mathrm{C}$, and supernatants were incubated with $15 \mu \mathrm{l}$ anti-GFP nanobody beads (KTSM1301, Shenzhen KT Life technology), anti-Flag conjugated agarose beads (M20018, Abmart) or anti-HA conjugated agarose beads (M20013, Abmart) for $1 \mathrm{~h}$ at $4{ }^{\circ} \mathrm{C}$. Beads were washed 3 times with wash buffer (10
$\mathrm{mM}$ Tris- $\mathrm{HCl} \mathrm{pH} 7.4,150 \mathrm{mM} \mathrm{NaCl}, 2.5 \mathrm{mM} \mathrm{MgCl} 2,10 \%$ Glycerol, $0.1 \% \mathrm{NP}-40$ ) and boiled in $20 \mu \mathrm{l} 2 \times \mathrm{SDS}$ buffer. Proteins were analyzed by immunoblotting. For immunoprecipitation from activated splenic B cells, cells were stimulated with LPS/IL4 for 3 days, collected, washed and processed as above.

\section{HTGTS}

HTGTS was performed as previously reported. ${ }^{65}$ In primary B cell CSR assay, $S$ region rearrangements were cloned from endogenous AID-initiated Sy1 breaks with $5^{\prime}$-RED-l $\mu$ primer. ${ }^{4}$ The HTGTS cloning primers are listed in Supplementary information, Table S2. $S$ junction and resection ratios were plotted and calculated as previously described. ${ }^{4}$

Transcription analyses

RNA-seq and data analysis

Prepare RNA-Seq library: Total RNA was isolated using Trizol reagents (15596018; Life Technologies) and was treated with DNase I (M6101; Promega). RNA-seq libraries were prepared using KAPA stranded RNA-Seq kits with RiboErase (HMR) (KR1142; KAPA) following the manufacturer's instructions. Then these libraries were sequenced with Illumina HiSeq at Geneseeq company. About $\sim 25$ million of $2 \times 150 \mathrm{bp}$ paired reads were retrieved for each library. Two biological replicates of mouse naive and CSRactivated $\mathrm{B}$ cells were performed for each genotype. Two repeats were performed in parental $\mathrm{CH} 12 \mathrm{~F} 3$ cell line along with two Ercc6/2 $2^{-1-}$ clones. One repeat was performed in parental U2OS cell line along with its isogenic Ercc6/2 ${ }^{-/-}$cell line.

Genome-wide analysis: The raw reads were first cleaned with cutadapt (v. 1.14) cut adapter sequences and mapped to mouse or human rRNA reference using bowtie2 (v. 2.2.6) to filter out rRNA reads. Then the filtered reads were mapped to mouse $(\mathrm{mm} 10)$ or human (hg38) reference genome with STAR (v. 2.5.3a) using default parameters. HTseq (v. 0.9.1) and DESeq2 (v. 1.14.1) were used for differential gene expression analysis. Package bamCoverage from deeptools ( $v$. 2.5.3) was used to generate bigwig files from bam files and IGV was used for downstream visualization.

\section{$P R O$-seq and data analysis}

Prepare PRO-Seq library: PRO-Seq was performed according to previously published protocol. ${ }^{66}$ For primary B cells, WT and Ercc $6 / 2^{-1-}$ B cells were harvested at $60 \mathrm{~h}$ after CSR-activation, and three biological replicates of WT B cells and four biological replicates of Ercc6/2 ${ }^{-/-}$B cells were subjected to PRO-Seq.

Data analysis: PRO-Seq data were first cut to remove adapter sequences with cutadapt (v. 1.14), then mapped to mouse reference genome $(\mathrm{mm} 10)$ by bowtie2 (v. 2.2.6). Transcribed regions were defined as previously described. Only coordinate of the last base at $3^{\prime}$ end of each read was exacted for pausing index calculation. Pausing index was defined as previously described. Briefly, we calculate the ratio of mean counts between -30 bp to $300 \mathrm{bp}$ relative to the TSS and the remaining length of the gene for NCBI RefSeq genes longer than $1 \mathrm{~kb}$.

Quantification and statistical analysis

Statistical analyses were performed by using the R (Version 3.5.1, R Foundation for Statistical Computing, Vienna, Austria. URL http:// www.R-project.org) or GraphPad Prism (version 8.0.1). The number of replicates, statistical test procedures are indicated in the figure legends.

\section{DATA AVAILABILITY}

All sequencing data generated in this study, including CRISPR screening, RNA-Seq, Pro-Seq, SHM amplicon-seq and HTGTS data, are deposited in the NCBI Sequence 
Read Archive (SRA accession: PRJNA624711). All other data are available from the authors on request.

\section{ACKNOWLEDGEMENTS}

We thank Drs Frederick Alt, Klaus Rajewsky, Tasuku Honjo, Kefei Yu, Guoliang Xu, Ronggui Hu, Yin Jing, Lan Wang, Jumin Zhou, Matthew Weitzman, Ming-Han Tong, Jun Huang and Yu Zhang for providing reagents, and Drs Jin-Qiu Zhou and Bin-Bing Zhou for critical reading of the manuscript, and Dr Daniel Durocher for communicating unpublished observations. We thank the Genome Tagging Project (GTP) Center, SIBCB, CAS for generating Ercc6/2-HA mouse model. This work was supported by National Key R\&D Program of China (2017YFA0506700, 2017YFC0908500), Strategic Priority Research Program of Chinese Academy of Sciences (XDB19030000), and National Natural Science Foundation of China (81622022, 31670929, 31800766). X.L. is supported by China Postdoctoral Science Foundation Grants (2018M632180, 2019T120363). GTP center is supported by a Shanghai Municipal Commission for Science and Technology Grant (17411954900).

\section{AUTHOR CONTRIBUTIONS}

F.L.M. conceived of the experiments. F.L.M., X.L., T.L., Y.S. and P.D. analyzed the data and prepared figures, with contributions from W.Z., B.J.L., X.Z. and S.Z. Experiments were performed by X.L., T.L., Y.S., P.D., M.H., B.J.L., D.Y. and L.D.L. W.Z., Q.W., T.X. and X. S.L. contributed to CRISPR screen design and data analyses. B.O.Z., J.D., L.S.Y. and J.H. provided essential materials and/or analysis tools. F.L.M., R.C., X.S.L., T.L., and Y.S. wrote the paper.

\section{ADDITIONAL INFORMATION}

Supplementary information accompanies this paper at https://doi.org/10.1038/ s41422-020-0328-3.

Competing interests: The authors declare no competing interests.

\section{REFERENCES}

1. Alt, F. W., Zhang, Y., Meng, F. L., Guo, C. \& Schwer, B. Mechanisms of programmed DNA lesions and genomic instability in the immune system. Cell 152, 417-429 (2013).

2. Schatz, D. G. \& Swanson, P. C. V(D)J recombination: mechanisms of initiation Annu. Rev. Genet. 45, 167-202 (2011).

3. Casellas, R. et al. Mutations, kataegis and translocations in B cells: understanding AID promiscuous activity. Nat. Rev. Immunol. 16, 164-176 (2016).

4. Dong, J. et al. Orientation-specific joining of AID-initiated DNA breaks promotes antibody class switching. Nature 525, 134-139 (2015).

5. Frock, R. L. et al. Genome-wide detection of DNA double-stranded breaks induced by engineered nucleases. Nat. Biotechnol. 33, 179-186 (2015).

6. $\mathrm{Hu}, \mathrm{J}$. et al. Chromosomal loop domains direct the recombination of antigen receptor genes. Cell 163, 947-959 (2015).

7. Stracker, T. H. \& Petrini, J. H. The MRE11 complex: starting from the ends. Nat. Rev. Mol. Cell Biol. 12, 90-103 (2011)

8. Panier, S. \& Boulton, S. J. Double-strand break repair: 53BP1 comes into focus Nat. Rev. Mol. Cell Biol. 15, 7-18 (2014).

9. Lieber, M. R. The mechanism of double-strand DNA break repair by the nonhomologous DNA end-joining pathway. Annu. Rev. Biochem. 79, 181-211 (2010).

10. Arnoult, N. et al. Regulation of DNA repair pathway choice in $\mathrm{S}$ and $\mathrm{G} 2$ phases by the NHEJ inhibitor CYREN. Nature 549, 548-552 (2017).

11. Hung, P. J. et al. MRI is a DNA damage response adaptor during classical nonhomologous end joining. Mol. Cell 71, 332-342 (2018).

12. Ochi, T. et al. DNA repair. PAXX, a paralog of XRCC4 and XLF, interacts with Ku to promote DNA double-strand break repair. Science 347, 185-188 (2015)

13. Zhang, Y. et al. The fundamental role of chromatin loop extrusion in physiological $\mathrm{V}(\mathrm{D}) \mathrm{J}$ recombination. Nature 573, 600-604 (2019).

14. Vian, L. et al. The energetics and physiological impact of cohesin extrusion. Cell 175, 292-294 (2018).

15. Zhang, X. et al. Fundamental roles of chromatin loop extrusion in antibody class switching. Nature 575, 385-389 (2019).

16. Yeap, L. S. \& Meng, F. L. Cis- and trans-factors affecting AID targeting and mutagenic outcomes in antibody diversification. Adv. Immunol. 141, 51-103 (2019).

17. Bunting, S. F. et al. 53BP1 inhibits homologous recombination in Brca1-deficient cells by blocking resection of DNA breaks. Cell 141, 243-254 (2010).
18. Panchakshari, R. A. et al. DNA double-strand break response factors influence end-joining features of IgH class switch and general translocation junctions. Proc. Natl. Acad. Sci. USA 115, 762-767 (2018).

19. $\mathrm{Li}, \mathrm{W}$. et al. MAGeCK enables robust identification of essential genes from genome-scale CRISPR/Cas9 knockout screens. Genome Biol. 15, 554 (2014).

20. Murai, J. et al. Trapping of PARP1 and PARP2 by clinical PARP inhibitors. Cancer Res. 72, 5588-5599 (2012).

21. Pommier, Y. DNA topoisomerase I inhibitors: chemistry, biology, and interfacial inhibition. Chem. Rev. 109, 2894-2902 (2009).

22. Rodriguez, R. et al. Small-molecule-induced DNA damage identifies alternative DNA structures in human genes. Nat. Chem. Biol. 8, 301-310 (2012).

23. Nitiss, J. L. Targeting DNA topoisomerase II in cancer chemotherapy. Nat. Rev. Cancer 9, 338-350 (2009).

24. Benitez-Bribiesca, L. \& Sanchez-Suarez, P. Oxidative damage, bleomycin, and gamma radiation induce different types of DNA strand breaks in normal lymphocytes and thymocytes. A comet assay study. Ann. N. Y. Acad. Sci. 887, 133-149 (1999).

25. Lundin, C. et al. Methyl methanesulfonate (MMS) produces heat-labile DNA damage but no detectable in vivo DNA double-strand breaks. Nucleic Acids Res. 33, 3799-3811 (2005)

26. Tummala, $H$. et al. ERCC6L2 mutations link a distinct bone-marrow-failure syndrome to DNA repair and mitochondrial function. Am. J. Hum. Genet. 94, 246-256 (2014).

27. Zhang, S. et al. A nonsense mutation in the DNA repair factor Hebo causes mild bone marrow failure and microcephaly. J. Exp. Med. 213, 1011-1028 (2016).

28. Bluteau, O. et al. A landscape of germ line mutations in a cohort of inherited bone marrow failure patients. Blood 131, 717-732 (2018).

29. Jarviaho, T. et al. Bone marrow failure syndrome caused by homozygous frameshift mutation in the ERCC6L2 gene. Clin. Genet. 93, 392-395 (2018).

30. Shabanova, I. et al. ERCC6L2-associated inherited bone marrow failure syndrome Mol. Genet. Genom. Med. 6, 463-468 (2018).

31. Methot, S. P. \& Di Noia, J. M. Molecular mechanisms of somatic hypermutation and class switch recombination. Adv. Immunol. 133, 37-87 (2017).

32. Han, L. \& Yu, K. Altered kinetics of nonhomologous end joining and class switch recombination in ligase IV-deficient B cells. J. Exp. Med. 205, 2745-2753 (2008).

33. Sonoda, E. et al. B cell development under the condition of allelic inclusion. Immunity 6, 225-233 (1997).

34. Pelanda, R. et al. Receptor editing in a transgenic mouse model: site, efficiency, and role in B cell tolerance and antibody diversification. Immunity 7, 765-775 (1997).

35. Tummala, H. et al. Genome instability is a consequence of transcription deficiency in patients with bone marrow failure harboring biallelic ERCC6L2 variants. Proc. Natl. Acad. Sci. USA 115, 7777-7782 (2018).

36. Rolland, T. et al. A proteome-scale map of the human interactome network. Cell 159, 1212-1226 (2014).

37. Helmink, B. A. \& Sleckman, B. P. The response to and repair of RAG-mediated DNA double-strand breaks. Annu. Rev. Immunol. 30, 175-202 (2012).

38. Kumar, V., Alt, F. W. \& Oksenych, V. Functional overlaps between XLF and the ATM-dependent DNA double strand break response. DNA Repair 16, 11-22 (2014).

39. Liu, X., Shao, Z., Jiang, W., Lee, B. J. \& Zha, S. PAXX promotes KU accumulation at DNA breaks and is essential for end-joining in XLF-deficient mice. Nat. Commun. 8, 13816 (2017).

40. Hung, P. J. et al. Deficiency of XLF and PAXX prevents DNA double-strand break repair by non-homologous end joining in lymphocytes. Cell Cycle 16, 286-295 (2017).

41. Tadi, S. K. et al. PAXX is an accessory c-NHEJ factor that associates with Ku70 and has overlapping functions with XLF. Cell Rep. 17, 541-555 (2016).

42. Lescale, C. et al. Specific roles of XRCC4 paralogs PAXX and XLF during V(D)J recombination. Cell Rep. 16, 2967-2979 (2016)

43. Kumar, V., Alt, F. W. \& Frock, R. L. PAXX and XLF DNA repair factors are functionally redundant in joining DNA breaks in a G1-arrested progenitor B-cell line. Proc. Natl Acad. Sci. USA 113, 10619-10624 (2016).

44. Balmus, G. et al. Synthetic lethality between PAXX and XLF in mammalian development. Genes Dev. 30, 2152-2157 (2016).

45. Xiao, A. et al. WSTF regulates the H2A.X DNA damage response via a novel tyrosine kinase activity. Nature 457, 57-62 (2009).

46. Yu, K., Chedin, F., Hsieh, C. L., Wilson, T. E. \& Lieber, M. R. R-loops at immunoglobulin class switch regions in the chromosomes of stimulated B cells. Nat. Immunol. 4, 442-451 (2003).

47. Zheng, $S$. et al. Non-coding RNA generated following lariat debranching mediates targeting of AID to DNA. Cell 161, 762-773 (2015).

48. Pavri, R. et al. Activation-induced cytidine deaminase targets DNA at sites of RNA polymerase II stalling by interaction with Spt5. Cell 143, 122-133 (2010). 
744

49. Biebricher, A. et al. PICH: a DNA translocase specially adapted for processing anaphase bridge DNA. Mol. Cell 51, 691-701 (2013).

50. Ke, Y. et al. PICH and BLM limit histone association with anaphase centromeric DNA threads and promote their resolution. EMBO J. 30, 3309-3321 (2011).

51. $\mathrm{Xu}$, J. et al. Structural basis for the initiation of eukaryotic transcription-coupled DNA repair. Nature 551, 653-657 (2017).

52. Brouwer, I. et al. Sliding sleeves of XRCC4-XLF bridge DNA and connect fragments of broken DNA. Nature 535, 566-569 (2016).

53. Langevin, F., Crossan, G. P., Rosado, I. V., Arends, M. J. \& Patel, K. J. Fancd2 counteracts the toxic effects of naturally produced aldehydes in mice. Nature 475, 53-58 (2011).

54. Canela, A. et al. Genome organization drives chromosome fragility. Cell 170, 507-521 (2017).

55. Madabhushi, R. et al. Activity-induced DNA breaks govern the expression of neuronal early-response genes. Cell 161, 1592-1605 (2015).

56. Ward, I. M. et al. 53BP1 is required for class switch recombination. J. Cell Biol. 165 , 459-464 (2004)

57. Zha, S. et al. ATM damage response and XLF repair factor are functionally redundant in joining DNA breaks. Nature 469, 250-254 (2011).

58. Cao, Q. et al. CRISPR-FOCUS: a web server for designing focused CRISPR screening experiments. PLoS One 12, e0184281 (2017).
59. Sanjana, N. E., Shalem, O. \& Zhang, F. Improved vectors and genome-wide libraries for CRISPR screening. Nat. Methods 11, 783-784 (2014).

60. Meng, F. L. et al. Convergent transcription atintragenic super-enhancers targets AID-initiated genomic instability. Cell 159, 1538-1548 (2014).

61. Jolly, C. J., Klix, N. \& Neuberger, M. S. Rapid methods for the analysis of immunoglobulin gene hypermutation: application to transgenic and gene targeted mice. Nucleic Acids Res. 25, 1913-1919 (1997).

62. Xiang, Y. \& Garrard, W. T. The downstream transcriptional enhancer, Ed, positively regulates mouse Ig kappa gene expression and somatic hypermutation. J. Immunol. 180, 6725-6732 (2008).

63. Yeap, L. S. et al. Sequence-intrinsic mechanisms that target AID mutational outcomes on antibody genes. Cell 163, 1124-1137 (2015).

64. $\mathrm{Xu}, \mathrm{H}$. et al. Sequence determinants of improved CRISPR sgRNA design. Genome Res. 25, 1147-1157 (2015).

65. Hu, J. et al. Detecting DNA double-stranded breaks in mammalian genomes by linear amplification-mediated high-throughput genome-wide translocation sequencing. Nat. Protoc. 11, 853-871 (2016).

66. Mahat, D. B. et al. Base-pair-resolution genome-wide mapping of active RNA polymerases using precision nuclear run-on (PRO-seq). Nat. Protoc. 11, 1455-1476 (2016) 